\title{
Salpingectomy before assisted reproductive technologies: a systematic literature review
}

\author{
Marco Noventa', Salvatore Gizzo ${ }^{1}$, Carlo Saccardi', Shara Borgato ${ }^{1}$, Amerigo Vitagliano ${ }^{1}$, Michela Quaranta², \\ Pietro Litta', Michele Gangemi', Guido Ambrosini ${ }^{1}$, Donato D'Antona ${ }^{1}$ and Stefano Palomba ${ }^{3 *}$
}

\begin{abstract}
Salpingectomy is largely used in case of hydrosalpinx in infertile women scheduled for assisted reproductive technologies (ART), whereas there is no consensus on its role in absence of hydrosalpinx. The current is a systematic literature review to collate all available evidence regarding salpingectomy as fertility enhancement procedure before ART in infertile patients. Our primary endpoint was to assess the impact of the surgical procedure on ovarian reserve, and secondary outcomes were to evaluate its benefits and harms on ART outcomes. We identified 29 papers of which 16 reporting data on the impact of tubal surgery on ovarian reserve and 24 (11 previously included) on ART outcomes. Available data suggested an absence of variation in ovarian reserve markers after unilateral salpingectomy while contradictory results were reported for bilateral surgery. Considering ART outcomes, data reported a significant improvement in ongoing pregnancy/live-birth rate in treated subjects without significant reduction in ovarian response to gonadotropin stimulation. In case of tubal disease, a surgical approach based on unilateral salpingectomy may be considered safe, without negative effects on ovarian reserve and ovarian response to controlled ovarian stimulation whilst having a positive effect on pregnancy rate. Data regarding bilateral salpingectomy and ovarian reserve are conflicting. Further trials are needed to confirm both the benefits of salpingectomy before ART and the safety of bilateral salpingectomy on ovarian reserve, and to clarify the role of uni- or bilateral surgery in case of tubal blockage without hydrosalpinx.
\end{abstract}

Keywords: Assisted reproduction, Hydrosalpinx, Infertility, Ovarian reserve, Salpingectomy

\section{Background}

Outstanding advances in technology have made in vitro fertilization (IVF) an everyday treatment for infertility. The rapid development of IVF and embryo transfer (ET) has seen assisted reproduction proposed as a valid choice for women affected by different type of infertility ranging from aging related problems [1-3] to organic pathologies such as tubal factor [4-6].

Assisted reproduction disregards the physiological role of salpinx during reproduction and has thus generated a minimal or absent interest for tubal diseases in women referred to IVF, a fact confirmed by the paucity of evidences available on this topic if compared with a large amount of literature continuously produced on

\footnotetext{
* Correspondence: stefanopalomba@tin.it

${ }^{3}$ Unit of Reproductive Surgery and Medicine, ASMN-IRCCS of Reggio Emilia, Reggio Emilia, Italy

Full list of author information is available at the end of the article
}

ovarian response, oocytes/embryo quality and endometrial factor. However, it has been demonstrated that, in patients suffering from hydrosalpinx and tubal factor infertility, the overall success of IVF is lower than expected due to implantation failure, miscarriage and ectopic pregnancy $[6,8]$.

These findings raised speculations regarding the detrimental role of salpinx fluid by both directly by contaminating the uterine cavity (reduction in embryo implantation) [9-11] and/or indirectly by exerting a toxic effect on the implanted embryo (impairment in embryo development) $[12,13]$. This led the United Kingdom's National institute of Health and Clinical Excellence guidelines (NICE) to recommend laparoscopic salpingectomy before assisted reproductive technologies (ART) in case of signs or suspicions of hydrosalpinx [14]. These Guidelines are also supported by data analyzed in a recent Cochrane review emphasizing a superior pregnancy rate when patients with tubal disease 
underwent laparoscopic salpingectomy (or at least tubal occlusion) prior to IVF treatments [15].

While salpingectomy before ART is universally recommended in case of evident hydrosalpinx [4, 5, 16, 17], no clear recommendations are available for the management of the wide spectrum of tubal pathologies of varying severity such as slight tubal dilatation (uni- or bilateral), previous tubal abortion and negative tubal patency test without hydrosalpinx. No clear recommendations regarding the surgical management of varying degrees of hydrosalpinx nor of unilateral hydrosalpinx with non-patent contralateral tube are available in the literature. The lack of accepted evidences regarding the potential detrimental effects of salpingectomy on ovarian reserve further complicate the determination of the most appropriate management for tubal disease before ART [18-20].

Based on these considerations, we performed a systematic literature review of all available evidence in order to summarize the actual benefits/harms of salpingectomy before ART in patients with and without hydrosalpinx.

\section{Methods}

A systematic literature search (English and French written literature) was conducted by electronic search of public databases MEDLINE, EMBASE, Science-Direct and the Cochrane library. We collected evidences dating from 1998 till 2015. This systematic review was conducted according to the Preferred Reporting Items for Systematic reviews and Meta-Analysis (PRISMA) guidelines. All longitudinal studies which evaluated ovarian reserve and subsequent ART outcomes following tubal surgery for enhancing fertility (i.e. unilateral and/or bilateral salpingectomy, tubal occlusion, etc.) were identified and analyzed. A manual search of the reference lists of the included studies and review articles was successively performed. All references of the retrieved studies were also reviewed to avoid overlooking relevant publications.

Primary outcome was to evaluate whether tubal surgery may have detrimental effects on ovarian reserve. Serum concentration of follicle stimulating hormone (FSH) and/or anti-Müllerian hormone (AMH) and/or antral follicle count (AFC) were considered as markers of ovarian reserve. Differences in ovarian reserve tests from baseline (pre-surgery) or in comparison with untreated (no surgery) arm were considered and discussed. Secondary outcome was to evaluate potential effects (benefits and harms) of tubal surgery on ovarian response in controlled stimulation cycles.

\section{Search strategy}

The key search terms included: "unilateral/bilateral salpingectomy" [Mesh] and "ovarian reserve" OR "AFC"

\section{OR "AMH” OR "FSH" OR "ART outcome" OR "IVF} outcome".

The selection of included studies was based on the availability of baseline AFC and/or baseline serum concentration of AMH and FSH in patients treated by unilateral/ bilateral salpingectomy (laparoscopy/laparotomy) for benign gynecological disease. We also included all studies which reported in detail ART outcomes in patients with a history of unilateral/bilateral salpingectomy (laparoscopy/ laparotomy) compared to untreated controls.

Titles and abstracts from electronic searches were scrutinized and reviewed by two authors independently (M.N; S.B) and full manuscripts and relative citation lists were analyzed by a third reviewer (S.G) with the scope of retrieving any omitted articles and selection of manuscripts by application of inclusion criteria.

Available data independently according to the subsequent topics: "Modifications in basal AMH and FSH after salpingectomy”, "ART after salpingectomy”, "ART outcomes following salpingectomy" were analyzed qualitatively.

\section{Inclusion and exclusion criteria}

Studies were considered eligible if they satisfied the following criteria: $i$ ) longitudinal prospective, randomized trial or retrospective studies; ii) study population comprised exclusively of women of reproductive age undergoing unilateral or bilateral salpingectomy for benign gynecological disease, $\mathrm{iii}$ ) presence of a control group either consisting of healthy matched controls or the same subjects prior to surgery; iv) ovarian reserve must be described by basal serum value of $\mathrm{AMH}$ and/or FSH and/or AFC for the entire sample; v) ART outcome must be described by at least one of the following, number of oocytes retrieved and/or number of obtained embryos and/or pregnancy rate. Exclusion criteria were considered: reviews and case report, duplicated data, and longitudinal studies referring to intrauterine insemination.

\section{Results}

The systematic literature search based on our pre-defined key search items yielded a total of 147 potentially relevant papers. Out of 147 only 29 met the inclusion criteria (Fig. 1) [1-13, 16-31]. A full report regarding authors, study design, sample size, type of surgical procedure (unilateral/bilateral salpingectomy), epidemiological features, surgical indications and years from surgery is illustrated in detail in Table 1.

The majority of patients underwent a laparoscopic surgical procedure (Table 1). The surgical indication was either hydrosalpinx or ectopic pregnancy in nearly all patients considered; only one manuscript included patients with salpingectomy due to tube-ovarian abscess [28]. Descriptive reports regarding baseline serum FSH 


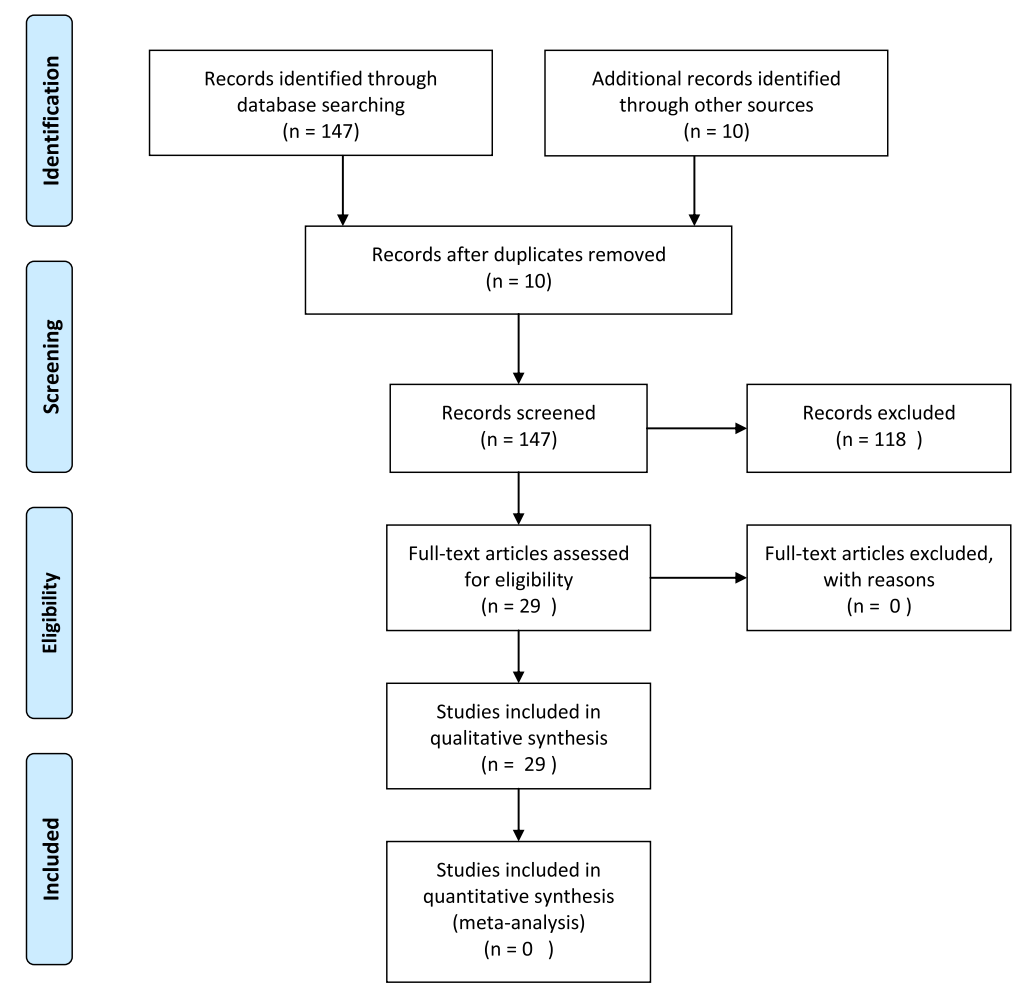

Fig. 1 Study' flow-chart according to PRISMA guidelines

and AMH serum values and AFC of patients included in the review are described in detail in Table 2. Descriptive reports concerning ART outcome [in particular: estradiol $\left(E_{2}\right)$ at ovulation induction, stimulation length, number of oocytes retrieved, obtained embryos, transferred embryos, implantation rate, clinical pregnancy rate, ongoing pregnancy rate] are reported in detail in Table 3.

\section{Effect on ovarian reserve markers}

\section{Basal FSH variations after uni/bilateral salpingectomy}

A total of twelve manuscripts reported evidence regarding possible variations in FSH serum values following uni/bilateral salpingectomy $[8,10,16,17,20-23,27-29$, 31]. We analyzed data from 1,569 patients. A total of 676 (43.1\%) patients underwent uni/bilateral salpingectomy, of these, $68.3 \%$ (462 patients) underwent unilateral salpingectomy and $20 \%$ (135 patients) bilateral salpingectomy. In a total of $79(11.7 \%)$ patients the technique was unspecified. Only $5.5 \%$ (86 women) of patients underwent proximal tubal division, ligation or occlusion. We excluded from data analysis $7(0.5 \%)$ patients from Uyar et al. [23] because they underwent surgical procedures other than salpingectomy or tubal division.

In particular, seven papers $[10,16,17,20,27,29,31]$ reported evidence from observational prospective studies evaluating FSH serum value before and after uni/bilateral salpingectomy. Six of these studies, including a total of 376 patients, did not report statistically significant differences in terms of serum FSH changes before and after surgical intervention [16, 17, 20, 27, 29, 31]. Only Gelbaya et al. [10] reported a significant increase in FSH after uni/bilateral salpingectomy in a total of 40 patients (detailed data are reported in Table 2).

The remaining five studies $[8,21-23,28]$ were observational and reported evidence from the comparison of patients treated by salpingectomy with untreated controls. In particular, four manuscripts, considering a total of 290 patients treated by unilateral/bilateral salpingectomy vs. 266 controls (50 proximal tubal division/ligation/occlusion, 56 sclero-therapy, 160 patients not treated surgically) did not reveal statistically significant differences in terms of serum FSH values [8, 21-23]. Only Ye et al. [28] reported a significant increase in FSH levels in 124 patients treated vs. 74 untreated controls (Table 2).

\section{Basal $A M H$ and $A F C$ variations after uni/bilateral salpingectomy}

Concerning basal AFC and AMH values we reported evidence from a total of nine studies $[13,18,22,23,25-28$, 31 . Four considered only basal AFC [13, 18, 23, 27], two only AMH [25] and three considered a combination of both parameters [22, 28, 31]. We analyzed data from 1,017 patients. A total of 510 (50.2 \%) underwent uni/ 
Table 1 Descriptive data of all eligible studies regarding patients, their general features, type and indication for surgery and type/duration of infertilitylncluded

\begin{tabular}{|c|c|c|c|c|c|c|c|c|c|c|}
\hline Authors \& Years & $\begin{array}{l}\text { Type of } \\
\text { Study }\end{array}$ & $\begin{array}{l}\text { Patients } \\
\text { (total) }\end{array}$ & $\begin{array}{l}\text { Patients } \\
\text { (Salpingectomy) }\end{array}$ & Patients-Controls & $\begin{array}{l}\text { Age } \\
(\text { year } \pm S D)\end{array}$ & BMI $( \pm$ SD) & $\begin{array}{l}\text { Indication for } \\
\text { Surgery }\end{array}$ & Time from Surgery & Reason of Infertility & $\begin{array}{l}\text { Duration of } \\
\text { Infertility }\end{array}$ \\
\hline Lass et al. [1] & $\mathrm{OP}$ & 102 & $\begin{array}{l}\text { a) } 29 \text { SLP (ECP) } \\
\text { Type: unilateral } \\
\text { Surgery: not } \\
\text { specified }\end{array}$ & $\begin{array}{l}\text { b) } 73 \text { no surgery } \\
\text { (healthy subjects) }\end{array}$ & $\begin{array}{l}\text { a) } 33.1 \pm 4.9 \\
\text { b) } 34.2 \pm 4.1\end{array}$ & $n . r$ & a) ECP & 2 years & a,b) MF 31; ID 42 & $\begin{array}{l}2 \text { years after } \\
\text { surgery at } \\
\text { least }\end{array}$ \\
\hline Dechaud et al. [2] & $\mathrm{RCT}$ & 60 & $\begin{array}{l}\text { a) } 30 \text { SLP (HY) } \\
\text { Type: not } \\
\text { specified } \\
\text { Surgery: LPS }\end{array}$ & $\begin{array}{l}\text { b) } 30 \text { diagnostic } \\
\text { LPS } \\
\text { (TF, HY) }\end{array}$ & $\begin{array}{l}\text { a) } 31.7 \pm 4.5 \\
\text { b) } 30.6 \pm 3.3\end{array}$ & $n \cdot r$ & $\begin{array}{l}\text { a,b) salpingitis } \\
\text { or } \mathrm{HY}\end{array}$ & $\begin{array}{l}\text { a) } 10.1 \pm 7.5 \text { months } \\
\text { b) } 9.5 \pm 7.2 \text { months } \\
\text { (LPS diagnostic) }\end{array}$ & $\mathrm{a}, \mathrm{b}) \mathrm{TF}$ & $\begin{array}{l}\text { a) } 55.2 \pm \\
33.3 \\
\text { months } \\
\text { b) } 48 \pm 25.4 \\
\text { months }\end{array}$ \\
\hline Bredkjaer et al. [3] & $\mathrm{R}$ & 278 & $\begin{array}{l}\text { a) } 139 \text { SLP (HY) } \\
\text { Type: } 128 \\
\text { bilateral, } 11 \text { partial } \\
\text { Surgery: not } \\
\text { specified }\end{array}$ & $\begin{array}{l}\text { b) } 139 \text { no surgery } \\
\text { (TF, no HY) }\end{array}$ & $\begin{array}{l}\text { a) } 32.6(22-39) \\
\text { b) } 32.9(24-40)\end{array}$ & $n . r$ & a) $\mathrm{HY}$ & $\begin{array}{l}92 \mathrm{pz} \text { between } 1.5 \\
\text { and } 5 \text { years } \\
47 \mathrm{pz} 3-6 \text { months }\end{array}$ & $\mathrm{a}, \mathrm{b}) \mathrm{TF}$ & $n . r$ \\
\hline Strandell et al. [4] & RCT & 204 & $\begin{array}{l}\text { a) } 116 \mathrm{SLP}(\mathrm{HY}) \\
\text { Type: not } \\
\text { specified } \\
\text { Surgery: LPS }\end{array}$ & $\begin{array}{l}\text { b) } 88 \text { no surgery } \\
\text { (TF, HY) }\end{array}$ & $\begin{array}{l}\text { a) } 31.8 \pm 3.6 \\
\text { b) } 31.8 \pm 3.7\end{array}$ & $n . r$ & $\begin{array}{l}\text { a,b) } \mathrm{HY} \text { uni o } \\
\text { bilateral }\end{array}$ & 2 months (at least) & $a, b) T F$ & $\begin{array}{l}1 \text { month to } \\
2 \text { years }\end{array}$ \\
\hline Dar et al. [5] & $\mathrm{R}$ & 26 & $\begin{array}{l}\text { a) } 26 \text { SLP (ECP) } \\
\text { Type : } 25 \\
\text { unilateral, } 1 \\
\text { bilateral } \\
\text { Surgery: LPS }\end{array}$ & $\begin{array}{l}\text { Control: same } \\
\text { patients before } \\
\text { surgery }\end{array}$ & a) $31.7 \pm 3.6$ & $n . r$ & a) ECP & $\begin{array}{l}\text { a period }<3 \text { years } \\
\text { between the cycles } \\
\text { (before and after } \\
\text { surgery) }\end{array}$ & $\begin{array}{l}\text { a) MEF 16; MF } 6 \\
\text { AN } 1 \text {; END } 1 \text {; ID } 2\end{array}$ & n.r \\
\hline Strandell et al. [6] & $\mathrm{OP}$ & 26 & $\begin{array}{l}\text { a) } 26 \text { SLP (HY) } \\
\text { Type: } 9 \text { unilateral } \\
\text { and } 17 \text { bilateral } \\
\text { Surgery: LPS }\end{array}$ & $\begin{array}{l}\text { Control: same } \\
\text { patients before } \\
\text { surgery }\end{array}$ & a) $32.7 \pm 3.6$ & $n . r$ & a) $\mathrm{HY}$ & n.r & $n . r$ & n.r \\
\hline Strandell et al. [7] & RCT & 185 & $\begin{array}{l}\text { a) } 103 \text { SLP (HY) } \\
\text { Type: } 40 \\
\text { unilateral, } 63 \\
\text { bilateral } \\
\text { Surgery: LPS }\end{array}$ & $\begin{array}{l}\text { b) } 82 \text { no surgery } \\
\text { (TF, HY) }\end{array}$ & $\begin{array}{l}\text { a) } 32.8 \pm 3.5 \\
\text { b) } 32.5 \pm 3.8\end{array}$ & $n . r$ & a) $\mathrm{HY}$ & $n . r$ & $a, b) T F$ & n.r \\
\hline Surrey et al. [8] & $\mathrm{R}$ & 94 & $\begin{array}{l}\text { a) } 32 \text { SLP (HY) } \\
\text { Type: not } \\
\text { specified } \\
\text { Surgery: LPS }\end{array}$ & $\begin{array}{l}\text { b) } 15 \text { PTO (HY) } \\
\text { c) } 35 \text { no surgery (TF } \\
\text { no HY) } \\
\text { d) } 12 \text { tubal ligation } \\
\text { (prior sterilization) }\end{array}$ & $\begin{array}{l}\text { a) } 35.1 \pm 0.7 \\
\text { b) } 35.4 \pm 1.0 \\
\text { c) } 35.6 \pm 0.7 \\
\text { d) } 38.2 \pm 1.0\end{array}$ & $n \cdot r$ & $\begin{array}{l}a, b) H Y \\
c, d) T F\end{array}$ & 6 months & $a, b, c, d) T F$ & n.r \\
\hline Tal et al. [12] & $\mathrm{OP}$ & 78 & $\begin{array}{l}\text { a) } 26 \text { SLP (ECP) } \\
\text { Type: unilateral } \\
\text { Surgery: } 14 \text { LPT, } \\
12 \text { LPS }\end{array}$ & $\begin{array}{l}\text { b) } 52 \text { no surgery } \\
\text { (healthy subjects) }\end{array}$ & $\begin{array}{l}\text { a) } 32.1 \pm 4.1 \\
\text { b) } 32.0 \pm 5.1\end{array}$ & $n . r$ & a) ECP & 1-9 years & $n \cdot r$ & n.r \\
\hline
\end{tabular}


Table 1 Descriptive data of all eligible studies regarding patients, their general features, type and indication for surgery and type/duration of infertilitylncluded (Continued)

\begin{tabular}{|c|c|c|c|c|c|c|c|c|c|c|}
\hline Chan et al. [13] & $R$ & 32 & $\begin{array}{l}32 \text { SLP (ECP) } \\
\text { a) } 18 \text { unilateral } \\
\text { LPS } \\
\text { b) } 14 \text { unilateral } \\
\text { LPT }\end{array}$ & $\begin{array}{l}\text { Controls: non- } \\
\text { operated site }\end{array}$ & $\begin{array}{l}\text { a) } 34(31-38) \\
\text { b) } 36(33-44)\end{array}$ & $\begin{array}{l}\text { a) } 20.96 \\
\text { b) } 21.44\end{array}$ & $a, b)$ ECP & 3 months (at least) & $n \cdot r$ & $n \cdot r$ \\
\hline Kontoravdis et al. [9] & $\mathrm{RCT}$ & 115 & $\begin{array}{l}\text { a) } 50 \text { POT (HY) } \\
\text { b) } 50 \mathrm{SLP}(\mathrm{HY}) \\
\text { Type: not } \\
\text { specified } \\
\text { Surgery: LPS }\end{array}$ & $\begin{array}{l}\text { c) } 15 \text { no surgery } \\
\text { (HY) }\end{array}$ & $\begin{array}{l}\text { a) } 31 \pm 4.5 \\
\text { b) } 29.8 \pm 3.4 \\
\text { c) } 34 \pm 5.3\end{array}$ & $n \cdot r$ & $\begin{array}{l}\text { a,b) } \mathrm{HY} \\
\text { unilateral or } \\
\text { bilateral }\end{array}$ & $n \cdot r$ & $n . r$ & n.r \\
\hline Gelbaya et al. [6] & $\mathrm{R}$ & 168 & $\begin{array}{l}\text { a) } 40 \text { SLP (HY) } \\
\text { Type: } 16 \\
\text { unilateral, } 24 \\
\text { bilateral } \\
\text { b) } 25 \text { PTD } \\
\text { Type: } 9 \text { unilateral, } \\
16 \text { bilateral } \\
\text { Surgery: LPS }\end{array}$ & $\begin{array}{l}\text { c) } 103 \text { no surgery } \\
\text { (tubal factor, no HY) }\end{array}$ & $\begin{array}{l}\text { a) } 32.8 \pm 3.57 \\
\text { b) } 33.1 \pm 2.71 \\
\text { c) } 33.5 \pm 3.32\end{array}$ & $n \cdot r$ & $a, b) H Y$ & 3 months at least & $\begin{array}{l}\text { All TF plus: } \\
\text { a) MF 2/40; OF 4/40; } \\
\text { END 2/40 } \\
\text { b) MF } 5 / 25 ; \text { OF } 5 / 25 ; \\
\text { END } 1 / 25 \\
\text { c) MF } 12 / 103 ; \text { OF } 9 / \\
\text { 103; END } 5 / 103\end{array}$ & n.r \\
\hline Moshin \& Hotineanu [11] & $\mathrm{RCT}$ & 204 & $\begin{array}{l}\text { a) } 60 \text { SLP (HY) } \\
\text { b) } 78 \text { PTO (HY) } \\
\text { Type: not } \\
\text { specified } \\
\text { Surgery: not } \\
\text { specified }\end{array}$ & $\begin{array}{l}\text { c) } 66 \text { no surgery } \\
\text { (HY) }\end{array}$ & n.r & $n \cdot r$ & $a, b) H Y$ & n.r & $n . r$ & n.r \\
\hline Sezik et al. [16] & $\mathrm{RCT}$ & 24 & $\begin{array}{l}\text { a) } 12 \text { total } \\
\text { hysterectomy + } \\
\text { bilateral- SLP } \\
\text { Surgery: LPT }\end{array}$ & $\begin{array}{l}\text { b) } 12 \text { total } \\
\text { hysterectomy } \\
\text { without SLP }\end{array}$ & $\begin{array}{l}\text { a) } 41.6 \pm 1.7 \\
\text { b) } 41.1 \pm 1.4\end{array}$ & $\begin{array}{l}\text { a) } 24.5 \pm 2.2 \\
\text { b) } 26.6 \pm 4.8\end{array}$ & n.r & n.r & $n \cdot r$ & n.r \\
\hline Nakagawa et al. [17] & $P$ & 17 & $\begin{array}{l}\text { a) } 6 \text { SLP (HY) } \\
\text { Type: not } \\
\text { specified } \\
\text { Surgery: LPS }\end{array}$ & $\begin{array}{l}\text { b) } 11 \text { PTD } \\
(\mathrm{HY})\end{array}$ & $\begin{array}{l}\text { a) } 31.7 \pm 6.3 \\
\text { b) } 35.3 \pm 3.6\end{array}$ & $n \cdot r$ & $a, b) H Y$ & $n \cdot r$ & $n . r$ & n.r \\
\hline Orvieto et al. [18] & $\mathrm{R}$ & 15 & $\begin{array}{l}\text { a) } 15 \text { SLP (HY) } \\
\text { Type: not } \\
\text { specified } \\
\text { Surgery: not } \\
\text { specified }\end{array}$ & $\begin{array}{l}\text { Controls: same } \\
\text { patients before } \\
\text { surgery }\end{array}$ & a) $32 \pm 4.4$ & $24 \pm 5.5$ & a) $\mathrm{HY}$ & n.r & $n . r$ & $n . r$ \\
\hline Almog et al. [19] & $\mathrm{R}$ & 36 & $\begin{array}{l}\text { a) } 36 \text { SLP } \\
\text { Type :22 } \\
\text { unilateral, } 14 \\
\text { bilateral } \\
\text { Surgery: LPS }\end{array}$ & $\begin{array}{l}\text { Controls: same } \\
\text { patients before } \\
\text { surgery }\end{array}$ & a) $34.2 \pm 4.5$ & $n \cdot r$ & $\begin{array}{l}\text { a) ECP } 21 \\
\text { HY } 14 \\
\text { Both } 1\end{array}$ & $152 \pm 36$ days & $n . r$ & n.r \\
\hline
\end{tabular}


Table 1 Descriptive data of all eligible studies regarding patients, their general features, type and indication for surgery and type/duration of infertilitylncluded (Continued)

\begin{tabular}{|c|c|c|c|c|c|c|c|c|c|c|}
\hline Xi et al. [20] & $\mathrm{R}$ & 156 & $\begin{array}{l}\text { a) } 76 \text { SLP (ECP) } \\
\text { Type: } 32 \text { bilateral, } \\
44 \text { unilateral ( } 23 \\
\text { controlateral } \\
\text { ligation) } \\
\text { Surgery: LPS }\end{array}$ & $\begin{array}{l}\text { Controls: same } \\
\text { patients before } \\
\text { surgery } \\
\text { b) } 80 \text { no surgery } \\
\text { (healthy subjects) }\end{array}$ & a) $31.5 \pm 4.2$ & $n \cdot r$ & a) ECP & $\begin{array}{l}\text { At least } 3 \text { months } \\
\text { after salpingectomy }\end{array}$ & $\begin{array}{l}\text { a) TF } 54 \\
\text { MF } 7 \\
\text { END } 5\end{array}$ & n.r \\
\hline Na et al. [21] & $\mathrm{R}$ & 97 & $\begin{array}{l}\text { a) } 41 \text { SLP (HY) } \\
\text { Type: not } \\
\text { specified } \\
\text { Surgery: LPS }\end{array}$ & $\begin{array}{l}\text { b) } 56 \text { sclerotherapy } \\
\text { (HY) }\end{array}$ & $\begin{array}{l}\text { a) } 32.4 \pm 4.5 \\
\text { b) } 32.9 \pm 4.1\end{array}$ & $\begin{array}{l}\text { a) } 22.2 \pm 5.0 \\
\text { b) } 21.5 \pm 2.2\end{array}$ & a, b) $H Y$ & n.r & $a, b) H Y$ & $\begin{array}{l}\text { a) } 3.8 \pm 3.4 \\
\text { b) } 2.9 \pm 1.8\end{array}$ \\
\hline Ni et al.[22] & PC & 134 & $\begin{array}{l}60 \text { SLP } \\
\text { a) } 26 \text { bilateral } \\
\text { b) } 34 \text { unilateral } \\
\text { c) } 23 \text { PTO } \\
\text { Surgery: LPS }\end{array}$ & $\begin{array}{l}\text { d) } 51 \text { no surgery } \\
\text { (TF, no HY) }\end{array}$ & $\begin{array}{l}\text { a) } 29.23 \pm 2.98 \\
\text { b) } 30.12 \pm 3.73 \\
\text { c) } 30.65 \pm 3.32 \\
\text { d) } 29.18 \pm 3.36\end{array}$ & $\begin{array}{l}\text { a) } 21.21 \pm 2.05 \\
\text { b) } 21.37 \pm 1.89 \\
\text { c) } 20.78 \pm 2.04 \\
\text { d) } 20.95 \pm 1.66\end{array}$ & $\begin{array}{l}\text { a, b) ECP, HY } \\
\text { c) } \mathrm{HY}\end{array}$ & n.r & $\begin{array}{l}\text { TF } \\
\text { n.s }\end{array}$ & $\begin{array}{l}\text { a) } 2.00 \pm 1.67 \\
\text { years } \\
\text { b) } 3.14 \pm 2.12 \\
\text { c) } 4.61 \pm 2.81 \\
\text { d) } 3.98 \pm 2.44\end{array}$ \\
\hline Uyar et al. [23] & $\mathrm{OP}$ & 162 & $\begin{array}{l}\text { a) } 33 \text { patients } \\
\text { (ECP) } \\
-26 \text { unilateral } \\
\text { SLP } \\
-3 \\
\text { salpingostomy } \\
-1 \text { tubal milkink } \\
-1 \text { fimbriectomy } \\
-2 \text { tubal abortion } \\
\text { surgery: LPS/LPT }\end{array}$ & $\begin{array}{l}\text { b) } 49 \text { MTX (ECP) } \\
\text { c) } 80 \text { no surgery }\end{array}$ & $\begin{array}{l}\text { a) } 31.1 \pm 5.1 \\
\text { b) } 29.7 \pm 5.0 \\
\text { c) } 28.9 \pm 6.0\end{array}$ & $n \cdot r$ & $a, b)$ ECP & n.r & $n \cdot r$ & n.r \\
\hline Lin et al. [24] & $\mathrm{R}$ & $\begin{array}{l}288 \text { cycles } \\
\text { in } 251 \\
\text { women }\end{array}$ & $\begin{array}{l}\text { a) } 103 \text { cycles in } \\
96 \text { SLP Type: not } \\
\text { specified } \\
\text { Surgery: LPS }\end{array}$ & $\begin{array}{l}\text { b) } 185 \text { cycles in } 155 \\
\text { women (prior } \\
\text { sterilization, } \\
\text { tuboplasty, PTO) } \\
\text { TF infertility }\end{array}$ & $\begin{array}{l}\text { a) } 33.2 \pm 4.2 \\
\text { b) } 32.8 \pm 4.6\end{array}$ & $\begin{array}{l}\text { a) } 22.1 \pm 4.3 \\
\text { b) } 21.9 \pm 3.2\end{array}$ & a) $\mathrm{ECP}$ or $\mathrm{HY}$ & n.a & $a, b) T F$ & n.a \\
\hline Grynnerup et al. [25] & P-CS & 71 & $\begin{array}{l}\text { a) } 16 \text { SLP (HY) } \\
\text { Type: (uni/ } \\
\text { bilateral) } \\
\text { not specified } \\
\text { Surgery: LPS }\end{array}$ & $\begin{array}{l}\text { b) } 42 \text { no surgery } \\
\text { (TF, with or without } \\
\text { HY) } \\
\text { c) } 13 \text { no surgery } \\
\text { (unexplained } \\
\text { infertility, no HY) }\end{array}$ & $\begin{array}{l}\text { a) } 34(25-37) \\
\text { b) } 33(26-37) \\
\text { c) } 32(27-36)\end{array}$ & $n . r$ & a) $\mathrm{HY}$ & At least 2 months & $\begin{array}{l}\text { a) HY } 16 \\
\text { b) TF } 42 \\
\text { c) ID } 13\end{array}$ & $\begin{array}{l}\text { a) } 5 \text { years } \\
\text { b) } 4 \text { years } \\
\text { c) } 5 \text { years }\end{array}$ \\
\hline Findley et al. [26] & $\mathrm{RCT}$ & 30 & $\begin{array}{l}\text { a) } 15 \\
\text { hysterectomy }+ \\
\text { bilateral SLP } \\
\text { Surgery: LPS }\end{array}$ & $\begin{array}{l}\text { b) } 15 \text { hysterectomy } \\
\text { with no SLP } \\
\text { LPS }\end{array}$ & $\begin{array}{l}\text { a) } 36.6 \pm 4.5 \\
\text { b) } 37.8 \pm 5.0\end{array}$ & $\begin{array}{l}\text { a) } 34.4 \pm 6.8 \\
\text { b) } 38.1 \pm 10.7\end{array}$ & $\begin{array}{l}\text { a,b) benign } \\
\text { indications }\end{array}$ & n.r & $n \cdot r$ & n.r \\
\hline Hill et al. [27] & $\mathrm{R}$ & 189 & $\begin{array}{l}\text { a) } 36 \text { SLP (ECP) } \\
\text { Type: unilateral }\end{array}$ & $\begin{array}{l}\text { Controls: same } \\
\text { patients before } \\
\text { surgery }\end{array}$ & $\begin{array}{l}\text { a) } 35.8 \pm 4.3 \\
\text { b) } 34.3 \pm 4.5\end{array}$ & $n \cdot r$ & a,b) ECP & $n . r$ & $n . r$ & n.r \\
\hline
\end{tabular}


Table 1 Descriptive data of all eligible studies regarding patients, their general features, type and indication for surgery and type/duration of infertilitylncluded (Continued)

\begin{tabular}{|c|c|c|c|c|c|c|c|c|c|c|}
\hline & & & $\begin{array}{l}\text { Surgery: not } \\
\text { specified }\end{array}$ & b) 153 MTX (ECP) & & & & & & \\
\hline Ye et al. [28] & $\mathrm{R}$ & 198 & $\begin{array}{l}\text { 124 SLP (HY, ECP, } \\
\text { TOA) } \\
\text { a) } 83 \text { unilateral } \\
\text { b) } 41 \text { bilateral } \\
\text { Surgery: not } \\
\text { specified }\end{array}$ & $\begin{array}{l}\text { c) } 74 \text { no surgery } \\
\text { (infertility, no TF) }\end{array}$ & $\begin{array}{l}\text { a) } 33,02 \pm 4,66 \\
\text { b) } 33,58 \pm 3,95 \\
\text { c) } 33,8 \pm 4,67\end{array}$ & $\begin{array}{l}\text { a) } 21,63 \pm 2,46 \\
\text { b) } 21,1 \pm 2,85 \\
\text { c) } 21,43 \pm 2,83\end{array}$ & $\begin{array}{l}\text { a) ECP } 79 \\
\text { HY } 3 \\
\text { TOA } 1 \\
\text { b) ECP } 24 \\
\text { HY } 16 \\
\text { TOA } 1\end{array}$ & n.r & $\begin{array}{l}\text { a) MF } 45 \text {; } \\
\text { MF \&FF } 28 \\
\text { b) MF } 16 \\
\text { MF \& FF } 15 \\
\text { c) MF } 38 \\
\text { MF \& FF } 26 \\
\text { UNKNOWN } 2\end{array}$ & $\begin{array}{l}\text { a) } 0.31 \pm 1.13 \\
\mathrm{Pl} \\
2.85 \pm 2.81 \mathrm{SI} \\
\text { b) } 0.82 \pm 1.96 \\
\mathrm{Pl} \\
3.23 \pm 3.24 \mathrm{SI} \\
\text { c) } 3.6 \pm 4.15 \\
\mathrm{Pl} \\
2.36 \pm 3.32 \mathrm{SI}\end{array}$ \\
\hline Pereira et al. [29] & $\mathrm{R}$ & 144 & $\begin{array}{l}\text { a) } 37 \text { SLP (ECP) } \\
\text { Type: unilateral } \\
\text { Surgery: LPS } \\
\text { b) } 107 \text { MTX (ECP) }\end{array}$ & $\begin{array}{l}\text { Controls: same } \\
\text { patients before } \\
\text { surgery or MTX }\end{array}$ & $\begin{array}{l}\text { a) } 36.4 \pm 3.03 \\
\text { b) } 37.1 \pm 4.01\end{array}$ & $\begin{array}{l}\text { a) } 24 \pm 3.65 \\
\text { b) } 23.2 \pm 4.23\end{array}$ & $a, b) E C P$ & 12 months & $\begin{array}{l}\text { a) A } 31.8 \% \text {; TF } 3 \\
13.6 \% \text {; END } 9 \% \text {; MF } \\
18.1 \% ; \text { ID } 4.55 \% \text {; } \\
\text { Other } 18.1 \% \\
\text { b) AN } 32.9 \% ; \text { TF } \\
\text { 12.5 \%: END } 7.95 \% ; \\
\text { MF } 23.9 \% \text { ID } 5.68 \% \\
\text { Other } 15.9 \%\end{array}$ & n.r \\
\hline Odesjo et al. [30] & $\mathrm{R}$ & 153 & $\begin{array}{l}\text { a) } 118 \text { SLP (ECP) } \\
\text { Type: unilateral } \\
\text { Surgery: not } \\
\text { specified }\end{array}$ & $\begin{array}{l}\text { b) } 35 \text { unilateral } \\
\text { salpingotomy } \\
(E C P)\end{array}$ & $\begin{array}{l}\text { a) } 32.5 \pm 3.93 \\
\text { b) } 33.8 \pm 3.07\end{array}$ & $\begin{array}{l}\text { a) } 24.9 \pm 4.5 \\
\text { b) } 24 \pm 3.76\end{array}$ & $a, b) E C P$ & $\begin{array}{l}\text { a) } 3.11 \pm 2.90 \text { years } \\
\text { b) } 6.85 \pm 5.05 \text { years }\end{array}$ & $\begin{array}{l}\text { a) TF } 92 \text { ( } 78 \%) \\
\text { a) other reasons } 26 \\
\text { ( } 22 \%) \\
\text { b) TF } 24(70.6 \%) \\
\text { b) other reason } 10 \\
(29.4 \%)\end{array}$ & n.r \\
\hline Venturella et al. [31] & RCT & 186 & $\begin{array}{l}\text { a) } 91 \text { SLP } \\
\text { standard } \\
\text { b) } 95 \text { SLP wide* } \\
\text { Type: bilateral } \\
\text { surgery: LPS }\end{array}$ & $\begin{array}{l}\text { Controls: same } \\
\text { patients before } \\
\text { surgery }\end{array}$ & $\begin{array}{l}\text { a) } 41.16 \pm 5.33 \\
\text { b) } 41.56 \pm 5.45\end{array}$ & $n \cdot r$ & $\begin{array}{l}\text { a,b) } \\
\text { myomectomy, } \\
\text { tubal surgical } \\
\text { sterilization }\end{array}$ & n.a & n.a & n.a \\
\hline
\end{tabular}

SLP, salpingectomy; n.r, not reported; n.a, not applicable; ECP, ectopic pregnancy; HY, Hydrosalpinx; TOA, tubo-ovarian abscess; FF, female factor (n.s), TF, tubal factor; OF, ovarian factor; MF, male factor; END, Endometriosis; MEF, mechanical factor; AN, anovulation; ID, idiopathic; R, retrospective; OP, observational-prospective; RCT, randomized controlled trial; P-CS, prospective cross-sectional study; PC, prospective cohort study; LPS, laparoscopy; LPT, laparotomy; PTD, proximal tubal division; PTO, proximal tubal occlusion/ligation; MTX, methotrexate; PI, primary infertility; Sl, secondary infertility 
Table 2 Ovarian reserve test (AMH and AFC) and basal-FSH of patients included in the review

\begin{tabular}{|c|c|c|c|c|c|c|c|}
\hline \multicolumn{8}{|l|}{ OVARIAN RESERVE } \\
\hline Authors \& Years & Patients & $\mathrm{AMH} \pm \mathrm{SD}$ & $p$ & $\mathrm{AFC} \pm \mathrm{SD} /$ range & $p$ & $\mathrm{FSH} \pm \mathrm{SD} /$ range & $p$ \\
\hline Surrey et al. [8] & $\begin{array}{l}\text { a) } 32 \text { SLP (HY) } \\
\text { Type: not specified } \\
\text { b) } 15 \text { PTO (HY) } \\
\text { c) } 35 \text { no surgery (TF no HY) } \\
\text { d) } 12 \text { tubal ligation }\end{array}$ & n.r. & & n.r. & & $\begin{array}{l}\text { a) } 7.07 \pm 0.12 \\
\text { b) } 6.65 \pm 0.42 \\
\text { c) } 7.08 \pm 0.23 \\
\text { d) } 7.47 \pm 0.25\end{array}$ & ns \\
\hline Chan et al. [13] & $\begin{array}{l}\text { 32 SLP (ECP) } \\
\text { a) } 18 \text { unilateral LPS } \\
\text { b) } 14 \text { unilateral LPT }\end{array}$ & $n \cdot r$ & - & $\begin{array}{l}\text { Operated site } \\
\text { a) } 5.0(3.0-7.3) \\
\text { b) } 6.5(1.8-10.3) \\
\text { Non operated site } \\
\text { a) } 7.5(4.8-8.3) \\
\text { b) } 4.0(2.8-9.3)\end{array}$ & $\begin{array}{l}\text { a) } .05 \\
\text { b) } n s\end{array}$ & $n \cdot r$ & - \\
\hline Gelbaya et al. [10] & $\begin{array}{l}\text { a) } 40 \text { SLP (HY) } \\
\text { Type: } 16 \text { unilateral, } 24 \text { bilateral } \\
\text { b) } 25 \text { PTD } \\
\text { Type: } 9 \text { unilateral, } 16 \text { bilateral } \\
\text { c) } 103 \text { no surgery } \\
\text { (tubal factor, no HY) }\end{array}$ & $n \cdot r$ & - & $n \cdot r$ & - & $\begin{array}{l}\text { IU/L } \\
\text { a) } 6.8 \pm 1.3 \text { pre } \\
7.61 \pm 2.31 \text { post } \\
\text { b) } 6.4 \pm 1.5 \text { pre } \\
6.35 \pm 1.51 \text { post } \\
\text { c) } 6.6 \pm 2.3 \text { pre } \\
6.71 \pm 2.32 \text { post }\end{array}$ & $\begin{array}{l}\text { a) } .05 \\
\text { b) } n s \\
\text { c) } n s\end{array}$ \\
\hline Sezik et al. [16] & $\begin{array}{l}\text { a) } 12 \text { total hysterectomy }+ \\
\text { bilateral SLP } \\
\text { b) } 12 \text { total hysterectomy without } \\
\text { SLP }\end{array}$ & $n \cdot r$ & - & $n \cdot r$ & - & $\begin{array}{l}\text { a) Basal } 4.8 \pm 1.4 \\
1 \text { month } 4.2 \pm 1.6 \\
6 \text { months } 4.4 \pm 2.1 \\
\text { b) Basal } 5.9 \pm 1.6 \\
1 \text { month } 5.2 \pm 1.6 \\
6 \text { months } 5.5 \pm 1.2\end{array}$ & ns \\
\hline Nakagawa et al. [17] & $\begin{array}{l}\text { a) } 6 \text { SLP (HY) } \\
\text { Type: not specified } \\
\text { b) } 11 \text { PTD }(H Y)\end{array}$ & $n \cdot r$ & - & $n . r$ & - & $\begin{array}{l}I U / L \\
\text { Before surgery } \\
\text { a) } 8.0 \pm 2.9 \\
\text { b) } 6.8 \pm 1.1 \\
\text { After surgery } \\
\text { a) } 8.6 \pm 4.0 \\
\text { b) } 14.1 \pm 9.3\end{array}$ & $\begin{array}{l}\text { a) } n s \\
\text { b) } n s\end{array}$ \\
\hline Orvieto et al. [18] & $\begin{array}{l}\text { a) } 15 \text { uni/bilateral SLP } \\
\text { (HY) }\end{array}$ & $n \cdot r$ & - & $\begin{array}{l}\text { Before surgery } \\
\text { a) } 5.6 \pm 2.5 \\
\text { affected side } \\
11.4 \pm 4.5 \text { both } \\
\text { side } \\
\text { After surgery } \\
\text { a) } 4.7 \pm 2.3 \\
\text { affected side } \\
9.5 \pm 4.9 \text { both } \\
\text { side }\end{array}$ & a) .05 & $n \cdot r$ & - \\
\hline Xi et al. [20] & $\begin{array}{l}\text { a) } 76 \text { SLP (ECP) } \\
\text { - } 44 \text { unilateral } \\
-32 \text { bilateral } \\
\text { b) } 80 \text { no surgery (healthy } \\
\text { subjects) }\end{array}$ & $n . r$ & - & $n \cdot r$ & - & $\begin{array}{l}\text { IU/L } \\
\text { Before surgery } \\
\text { a) } 6.9 \pm 1.5 \\
7.5 \pm 1.5 \\
7.0 \pm 1.5 \\
\text { After surgery } \\
\text { a) } 7.2 \pm 1.6 \\
7.3 \pm 1.2 \\
7.2 \pm 1.4 \\
\text { b) } 7.2 \pm 1.8\end{array}$ & a) $n s$ \\
\hline Na et al. [21] & $\begin{array}{l}\text { a) } 41 \text { SLP (HY) } \\
\text { Type: not specified } \\
\text { b) } 56 \text { sclerotherapy (HY) }\end{array}$ & $n . r$ & - & $n . r$ & - & $\begin{array}{l}\text { IU/L } \\
\text { a) } 8.2 \pm 4.6 \\
\text { b) } 10.9 \pm 17.1\end{array}$ & ns \\
\hline Grynnerup et al. [25] & $\begin{array}{l}\text { a) } 16 \text { SLP (HY) } \\
\text { Type: not specified } \\
\text { b) } 42 \text { no surgery (TF, with or } \\
\text { without } \mathrm{HY} \text { ) }\end{array}$ & $\begin{array}{l}\text { pmol/L } \\
\text { a) } 16,1(5,2-54) \\
\text { b) } 23,4(3,5-50) \\
\text { c) } 21.8(12-64)\end{array}$ & $\begin{array}{l}\text { a) } n s \\
\text { b) } n s \\
\text { c) } n s\end{array}$ & $n \cdot r$ & - & $n \cdot r$ & - \\
\hline
\end{tabular}


Table 2 Ovarian reserve test (AMH and AFC) and basal-FSH of patients included in the review (Continued)

\begin{tabular}{|c|c|c|c|c|c|c|c|}
\hline Uyar et al. [23] & $\begin{array}{l}\text { a) } 33 \text { patients (ECP) } \\
\text { - } 26 \text { unilateral SLP } \\
-3 \text { salpingostomy } \\
\text { b) } 49 \text { MTX (ECP) } \\
\text { c) } 80 \text { no surgery }\end{array}$ & $n . r$ & - & $\begin{array}{l}\text { a) } 10.1 \pm 3.5 \\
\text { b) } 10.3 \pm 4.1 \\
\text { c) } 10.1 \pm 3.5\end{array}$ & $\begin{array}{l}\text { a) } n s \\
\text { b) } n s \\
\text { c) } n s\end{array}$ & $\begin{array}{l}\text { IU/L } \\
\text { a) } 7.7 \pm 3.8 \\
\text { b) } 7.7 \pm 2.3 \\
\text { c) } 7.9 \pm 1.9\end{array}$ & $\begin{array}{l}\text { a) } n s \\
\text { b) } n s \\
\text { c) } n s\end{array}$ \\
\hline Ni et al. [22] & $\begin{array}{l}60 \text { SLP (ECP, HY) } \\
\text { a) } 26 \text { bilateral } \\
\text { b) } 34 \text { unilateral } \\
\text { c) } 23 \text { PTO (HY) } \\
\text { d) } 51 \text { no surgery } \\
\text { (TF, no HY) }\end{array}$ & $\begin{array}{l}\mathrm{pg} / \mathrm{mL} \\
\text { a) } 90.00 \\
\text { b) } 100.00 \\
\text { c) } 100.00 \\
\text { d) } 110.00\end{array}$ & $\begin{array}{l}\text { a) } n s \\
\text { b) } n s \\
\text { c) } n s \\
\text { d) } n s\end{array}$ & $\begin{array}{l}\text { a) } 9 \\
\text { b) } 10 \\
\text { c) } 11 \\
\text { d) } 11\end{array}$ & $\begin{array}{l}\text { a) } n s \\
\text { b) } n s \\
\text { c) } n s \\
\text { d) } n s\end{array}$ & $\begin{array}{l}\text { IU/L } \\
\text { a) } 7.35 \pm 1.59 \\
\text { b) } 7.17 \pm 1.16 \\
\text { c) } 7.64 \pm 2.10 \\
\text { d) } 7.13 \pm 1.57\end{array}$ & $\begin{array}{l}\text { a) } n s \\
\text { b) } n s \\
\text { c) } n s \\
\text { d) } n s\end{array}$ \\
\hline Findley et al. [26] & $\begin{array}{l}\text { a) } 15 \text { hysterectomy + bilateral SLP } \\
\text { b) } 15 \text { hysterectomy with no SLP }\end{array}$ & $\begin{array}{l}\text { a) Basal } 2.26 \pm 2.72 \\
4-6 \text { weeks } 1.03 \pm 1.04 \\
3 \text { months } 1.86 \pm 1.99 \\
\text { b) Basal } 2.25 \pm 2.57 \\
4-6 \text { weeks } 1.25 \pm 2.09 \\
3 \text { months } 1.82 \pm 3.12\end{array}$ & ns & $n \cdot r$ & - & $n \cdot r$ & - \\
\hline Hill et al. [27] & $\begin{array}{l}\text { a) } 36 \text { SLP (ECP) } \\
\text { Type: unilateral } \\
\text { b) } 153 \text { MTX (ECP) }\end{array}$ & $n . r$ & - & $\begin{array}{l}\text { a) } 10(3-50) \text { pre } \\
10(4-45) \text { post } \\
\text { b) } 12(1-53) \text { pre } \\
13(1-60) \text { post }\end{array}$ & $\begin{array}{l}\text { a) } n s \\
\text { b) } n s\end{array}$ & $\begin{array}{l}\text { IU/L } \\
\text { a) } 7.2(2.6-16.0) \text { pre } \\
7.9(5.1-10.4) \text { post } \\
\text { b) } 6.9(2.4-14.2) \text { pre } \\
7.2(2.3-16.3) \text { post }\end{array}$ & $\begin{array}{l}\text { a) } n s \\
\text { b) } n s\end{array}$ \\
\hline Pereira et al. [29] & $\begin{array}{l}\text { a) } 37 \text { SLP (ECP) } \\
\text { Type: unilateral } \\
\text { b) } 107 \text { MTX (ECP) }\end{array}$ & $n \cdot r$ & - & $n . r$ & - & $\begin{array}{l}\mathrm{m} / \mathrm{U} / \mathrm{mL} \\
\text { a) } 4.98 \pm 2.19 \text { pre } \\
4.87 \pm 2.97 \text { post } \\
\text { b) } 4.81 \pm 2.75 \text { pre } \\
4.94 \pm 2.05 \text { post }\end{array}$ & $\begin{array}{l}\text { a) } n s \\
\text { b) } n s\end{array}$ \\
\hline Ye et al. [28] & $\begin{array}{l}124 \text { SLP (HY, ECP, TOA) } \\
\text { a) } 83 \text { unilateral } \\
\text { b) } 41 \text { bilateral } \\
\text { c) } 74 \text { no surgery (infertility, no TF) }\end{array}$ & $\begin{array}{l}\mathrm{fmol} / \mathrm{mL} \\
\text { a) } 167,56 \pm 127,03 \\
\text { b) } 127,11 \pm 93,23 \\
\text { c) } 183.48 \pm 104,37\end{array}$ & .05 & $\begin{array}{l}\text { a) } 10,7 \pm 3,62 \\
\text { b) } 9,58 \pm 3,73 \\
\text { c) } 11,2 \pm 4,16\end{array}$ & ns & $\begin{array}{l}\mathrm{mIU} / \mathrm{mL} \\
\text { a) } 8.42 \pm 2.3 \\
\text { b) } 9.13 \pm 3.2 \\
\text { c) } 7.85 \pm 2.69\end{array}$ & .05 \\
\hline Venturella et al. [31] & $\begin{array}{l}\text { a) } 91 \text { SLP standard } \\
\text { b) } 95 \text { SLP wide } \\
\text { Type: bilateral }\end{array}$ & $\begin{array}{l}\mathrm{ng} / \mathrm{mL} \\
\text { Before surgery } \\
\text { a) } 0.93 \pm 1.13 \\
\text { b) } 0.86 \pm 1.01 \\
\Delta \text { After surgery } \\
\text { a) }-0.09 \pm 0.24(\Delta) \\
\text { b) }-0.07 \pm 0.22(\Delta)\end{array}$ & $\begin{array}{l}\text { a) } n s \\
\text { b) } n s\end{array}$ & $\begin{array}{l}\text { Before surgery } \\
\text { a) } 7.8 \pm 4.23 \\
\text { b) } 6.82 \pm 4.68 \\
\Delta \text { After surgery } \\
\text { a) }-0.33 \pm 0.73(\Delta) \\
\text { b) }-0.26 \pm 0.61 \\
\text { ( } \Delta)\end{array}$ & $\begin{array}{l}\text { a) } n s \\
\text { b) } n s\end{array}$ & $\begin{array}{l}\mathrm{mIU} / \mathrm{mL} \\
\text { Before surgery } \\
\text { a) } 12.9 \pm 9.71 \\
\text { b) } 12.39 \pm 7.88 \\
\text { After surgery } \\
\text { a) } 0.47 \pm 0.86 \\
\text { b) } 0.37 \pm 0.84\end{array}$ & $\begin{array}{l}\text { a) } n s \\
\text { b) } n s\end{array}$ \\
\hline
\end{tabular}

n.r, not reported; ns, not significant; LPS, laparoscopy; LPT, laparotomy; MTX, methotrexate; PTD, proximal tubal division; PTO, Proximal tubal occlusion; ${ }^{\circ}$ On 21 patients (23 contro-lateral ligation excluded)

bilateral salpingectomy; of these, $75.1 \%$ (383 patients), underwent unilateral salpingectomy, $18.8 \%$ (96 patients) bilateral salpingectomy and $6.1 \%$ (31 patients) unspecified procedures. Only 23 (2.3\%) patients underwent tubal division. Three manuscripts, for a total of 237 (23.3\% of the sample) patients, defined the control group as the same cohort of patients prior to undergoing surgery [18, 27, 31]. Four manuscripts defined the control group as either untreated patients, for a total of $260(25.6 \%)$ or patients treated by methotrexate (MTX) for ectopic pregnancy, for a total of $202(19.9 \%)$ [22, 23, 25, 28]. Chan et al. [13] defined as the control group for AFC count, the counterlateral adnexa of the same group of patients treated by unilateral salpingectomy. Findley et al. [26] established as control group patients subjected to total hysterectomy without salpingectomy. We excluded from data analysis 7 $(0.7 \%)$ patients from Uyar et al. [23] because they underwent surgical procedures other than salpingectomy or tubal division.
In three studies $[18,27,31]$, the variation in basal AFC before and after unilateral/bilateral salpingectomy in the same cohort of patients was evaluated. Two, reporting evidence derived from observation of 222 patients, did not observe statistically significant differences in terms of AFC after unilateral salpingectomy $[27,31]$. Only one paper evaluating a total of 15 cases of uni/bilateral salpingectomy reported a significant decrease in AFC [18]. Three manuscripts reported evidence from observational studies comparing patients surgically treated versus controls (no-treated or medically MTX) In a total of 210 patients who underwent uni/bilateral salpingectomy compared to 49 patients treated by MTX for ectopic pregnancy, 23 patients who underwent tubal division and finally 205 not untreated patients, the three Authors did not observe any statistically significant decrease in AFC [22, 23, 28]. Only Chan et al. [13] noted significant differences in a cohort of 32 patients treated by unilateral laparoscopic and 
Table 3 IVF outcome of patients included in the review

\begin{tabular}{|c|c|c|c|c|c|c|c|c|c|c|c|c|c|c|c|c|c|}
\hline \multicolumn{18}{|c|}{ IVF TREATMENT } \\
\hline $\begin{array}{l}\text { Authors \& } \\
\text { Years }\end{array}$ & Patients & $\begin{array}{l}\text { E2 (on hCG day) } \\
\pm S D\end{array}$ & $p$ & $\begin{array}{l}\text { Stimulation Lenght } \\
\text { (Days } \pm \text { SD) }\end{array}$ & $p$ & $\begin{array}{l}\text { No. Oocytes } \\
\text { retrieved } \pm \text { SD/ } \\
\text { range }\end{array}$ & $p$ & $\begin{array}{l}\text { No. Obtained } \\
\text { Embryos } \pm \text { SD }\end{array}$ & $p$ & $\begin{array}{l}\text { No. transferred } \\
\text { Embryos } \pm S D / \text { range }\end{array}$ & $p$ & $\begin{array}{l}\text { Implantation } \\
\text { Rate \% (N) }\end{array}$ & $p$ & $\begin{array}{l}\text { Pregnancy } \\
\text { Rate \% (N) }\end{array}$ & $p$ & $\begin{array}{l}\text { Ongoing pregnancy } \\
\text { rate } \%(\mathrm{~N})\end{array}$ & $p$ \\
\hline $\begin{array}{l}\text { Lass et al. } \\
\text { [1] }\end{array}$ & $\begin{array}{l}\text { a) } 29 \text { SLP (ECP) } \\
\text { Type: unilateral } \\
\text { b) } 73 \text { no surgery } \\
\text { (healthy subjects) }\end{array}$ & $\begin{array}{l}\text { pmol/L } \\
\text { a) } 6.087 \pm 2.889 \\
\text { b) } 6.635 \pm 2735\end{array}$ & ns & $\begin{array}{l}\text { a) } 12.3 \pm 1.6 \\
\text { b) } 13.1 \pm 2.0\end{array}$ & ns & $\begin{array}{l}\text { - General } \\
\text { a) } 9.9 \pm 5.3 \\
\text { b) } 9.9 \pm 5.3 \\
\text { c) Ipsilateral } \\
\text { ovary } \\
3.8 \pm 3.0 \\
\text { Controlateral } \\
\text { ovary } \\
6.0 \pm 3.6\end{array}$ & $\begin{array}{l}\text { a, } \\
\text { b) } \\
\text { ns } \\
\text { c) } \\
.01\end{array}$ & $n \cdot r$ & - & $\begin{array}{l}\text { a) } 2.4 \pm 0.5 \\
\text { b) } 2.0 \pm 0.7\end{array}$ & ns & $n \cdot r$ & - & $\begin{array}{l}\text { a) } 17.2(5) \\
\text { b) } 13.7(10)\end{array}$ & ns & $n \cdot r$ & - \\
\hline $\begin{array}{l}\text { Dechaud et } \\
\text { al. [2] }\end{array}$ & $\begin{array}{l}\text { a) } 30 \text { SLP (HY) } \\
\text { Type: not specified } \\
\text { b) } 30 \text { no surgery } \\
\text { (TF, HY) }\end{array}$ & $\begin{array}{l}\mathrm{pmol} / \mathrm{L} \\
\text { a) } 2.699 \\
\text { b) } 1.903\end{array}$ & ns & $n \cdot r$ & - & $\begin{array}{l}\text { a) } 10.1 \pm 5.0 \\
\text { b) } 10.5 \pm 6.0\end{array}$ & ns & $\begin{array}{l}\text { a) } 5.2 \pm 3.4 \\
\text { b) } 4.8 \pm 3.7\end{array}$ & ns & $n \cdot r$ & - & $\begin{array}{l}\text { - First } \\
\text { attempts } \\
\text { a) } 10.4(5 / 48) \\
\text { b) } 4.6(2 / 43) \\
\text { - All attempts } \\
\text { a) } 13.4(21 / 156) \\
\text { b) } 8.6(10 / 116)\end{array}$ & ns & $\begin{array}{l}\text { - Per cycle } \\
\text { a) } 23.7(14 / 59) \\
\text { b) } 16.3(8 / 49) \\
\text { - Per oocyte } \\
\text { retrieval } \\
\text { a) } 31.8(14 / 44) \\
\text { b) } 23.5(8 / \\
\text { 34) } \\
\text { - Per transfer } \\
\text { a) } 36.8(14 / \\
\text { 38) } \\
\text { b) } 25(8 / 32)\end{array}$ & ns & $\begin{array}{l}\text { a) } 34.2(13 / 38) \\
\text { b) } 18.7(6 / 32)\end{array}$ & ns \\
\hline $\begin{array}{l}\text { Bredkjaer et } \\
\text { al. [3] }\end{array}$ & $\begin{array}{l}\text { a) } 139 \text { SLP (HY) } \\
\text { Type: } 128 \text { bilateral, } 11 \\
\text { partial } \\
\text { b) } 139 \text { no surgery } \\
\text { (TF, no HY) }\end{array}$ & $n . r$ & - & $n . r$ & & $\begin{array}{l}\text { a) } 9.3 \\
\text { b) } 9.1\end{array}$ & ns & $n . r$ & & $\begin{array}{l}\text { a) } 2.1 \\
\text { b) } 2.1\end{array}$ & ns & $\begin{array}{l}\text { a) } 19 \\
\text { b) } 21\end{array}$ & ns & $\begin{array}{l}\text { a) } 40.3(106) \\
\text { b) } 40.5(120)\end{array}$ & ns & $\begin{array}{l}\text { a) } 21.7(57) \\
\text { b) } 21.6(64)\end{array}$ & ns \\
\hline $\begin{array}{l}\text { Strandell et } \\
\text { al. [4] }\end{array}$ & $\begin{array}{l}\text { a) } 116 \text { SLP (HY) } \\
\text { Type: not specified } \\
\text { b) } 88 \text { no surgery } \\
\text { (TF, HY) }\end{array}$ & $n \cdot r$ & - & $\begin{array}{l}\text { a) } 11.4 \pm 2.2 \\
\text { b) } 11.6 \pm 2.9\end{array}$ & ns & $\begin{array}{l}\text { a) } 10.6 \pm 5.9 \\
\text { b) } 10.6 \pm 6.1\end{array}$ & ns & $\begin{array}{l}\text { a) } 6.8 \pm 4.8 \\
\text { b) } 7.0 \pm 4.9\end{array}$ & ns & $\begin{array}{l}\text { a) } 2.0 \pm 0.3 \\
\text { b) } 2.0 \pm 0.4\end{array}$ & ns & $\begin{array}{l}\text { - Per transfer } \\
\text { cycle } \\
\text { a) } 22.8(47 / 206) \\
\text { b) } 18.8(28 / 149) \\
\text { - Ultrasound } \\
\text { visible HY } \\
\text { a) } 30(21 / 70) \\
\text { b) } 16.7(13 / 78)\end{array}$ & $\begin{array}{l}\text { ns } \\
.05\end{array}$ & $\begin{array}{l}\text { a) } 36.6(41) \\
\text { b) } 23.9(22)\end{array}$ & .05 & $\begin{array}{l}\text { a) } 28.6(32) \\
\text { b) } 16.3(15)\end{array}$ & .05 \\
\hline Dar et al. [5] & $\begin{array}{l}\text { a) } 26 \text { SLP (ECP) } \\
\text { Type: } 25 \text { unilateral, } 1 \\
\text { bilateral } \\
\text { Control: same patients } \\
\text { before surgery }\end{array}$ & $n . r$ & - & $\begin{array}{l}\text { Before surgery } \\
\text { a) } 10.81 \pm 2.45 \\
\text { After Surgery } \\
\text { a) } 10.68 \pm 2.57\end{array}$ & ns & $\begin{array}{l}\text { Before surgery } \\
\text { a) - Operated site } \\
6.06 \pm 3.85 \\
\text { - Non operated } \\
\text { site } \\
5.07 \pm 3.08 \\
\text { After Surgery } \\
\text { a) - Operated site } \\
5.31 \pm 4.22 \\
\text { - Non operated } \\
\text { site } 4.4 \pm 3.68\end{array}$ & ns & $n \cdot r$ & - & $\begin{array}{l}\text { Before surgery } \\
\text { a) } 3.56 \pm 0.81 \\
\text { After Surgery } \\
\text { a) } 3.37 \pm 0.8\end{array}$ & ns & $\begin{array}{l}\text { After Surgery } \\
\text { a) } 23.07\end{array}$ & - & $\begin{array}{l}\text { After } \\
\text { Surgery } \\
\text { a) } 19.23\end{array}$ & - & $n \cdot r$ & - \\
\hline
\end{tabular}


Table 3 IVF outcome of patients included in the review (Continued)

\begin{tabular}{|c|c|c|c|c|c|c|c|c|c|c|c|c|c|c|c|c|c|}
\hline $\begin{array}{l}\text { Strandell et } \\
\text { al. [6] }\end{array}$ & $\begin{array}{l}\text { Before surgery } \\
\text { a) } 26 \text { SLP (general) } \\
\text { b) } 9 \text { unilateral } \\
\text { c) } 17 \text { bilateral } \\
\text { After Surgery } \\
\text { a) } 26 \text { SLP (general) } \\
\text { b) } 9 \text { unilateral } \\
\text { c) } 17 \text { bilateral }\end{array}$ & $n \cdot r$ & - & $\begin{array}{l}\text { Before surgery } \\
\text { a) } 11 \pm 2.4 \\
\text { b) } 12.2 \pm 2.4 \\
\text { c) } 10.4 \pm 2.3 \\
\text { After Surgery } \\
\text { a) } 11.2 \pm 2.3 \\
\text { b) } 12.2 \pm 2.4 \\
\text { c) } 10.6 \pm 2.1\end{array}$ & ns & $\begin{array}{l}\text { Before surgery } \\
\text { a) } 9.4 \pm 5.9 \\
\text { b) } 9.1 \pm 4.4 \\
\text { c) } 9.5 \pm 6.7 \\
\text { After Surgery } \\
\text { a) } 8.7 \pm 5.7 \\
\text { b) } 7.7 \pm 4.6 \\
\text { c) } 9.2 \pm 6.2\end{array}$ & ns & $\begin{array}{l}\text { Before } \\
\text { surgery } \\
\text { a) } 7.0 \pm 5.6 \\
\text { b) } 6.4 \pm 3.9 \\
\text { c) } 7.3 \pm 6.5 \\
\text { After Surgery } \\
\text { a) } 5.9 \pm 4.3 \\
\text { b) } 5.3 \pm 4.3 \\
\text { c) } 6.2 \pm 4.4\end{array}$ & $n s$ & $n . r$ & - & $n \cdot r$ & - & $n \cdot r$ & - & $n \cdot r$ & - \\
\hline $\begin{array}{l}\text { Strandell et } \\
\text { al. [7] }\end{array}$ & $\begin{array}{l}\text { a) } 103 \text { SLP (HY) } \\
\text { Type: } 40 \text { unilateral, } 63 \\
\text { bilateral } \\
\text { b) } 82 \text { no surgery } \\
\text { (TF, HY) }\end{array}$ & $n \cdot r$ & - & $\begin{array}{l}\text { a) } 11.3 \pm 2.1 \\
\text { b) } 11.4 \pm 2.6\end{array}$ & ns & $\begin{array}{l}\text { a) } 10.3 \pm 5.4 \\
\text { b) } 10.6 \pm 5.4\end{array}$ & ns & $\begin{array}{l}\text { a) } 6.8 \pm 4.1 \\
\text { b) } 7.1 \pm 4.4\end{array}$ & ns & $\begin{array}{l}\text { a) } 2.0 \pm 0.3 \\
\text { b) } 2.0 \pm 0.3\end{array}$ & ns & $n \cdot r$ & - & $\begin{array}{l}\text { ODD RATIO } \\
\text { - Total } \\
\text { group } 1.7 \\
\text { - US visible } \\
\text { HY } 2.8 \\
\text { - US visible } \\
\text { HY bilateral } \\
6.9\end{array}$ & .05 & $\begin{array}{l}\text { HAZARD R (BR) } \\
\text { - Total group } 2.1 \\
\text { - US visible HY } 3.8 \\
\text { US visible HY } \\
\text { bilateral } 6.0\end{array}$ & .05 \\
\hline $\begin{array}{l}\text { Surrey et al. } \\
\text { [8] }\end{array}$ & $\begin{array}{l}\text { a) } 32 \text { SLP (HY) } \\
\text { Type: not specified } \\
\text { b) } 15 \text { PTO (HY) } \\
\text { c) } 35 \text { no surgery (TF } \\
\text { no HY) } \\
\text { d) } 12 \text { PTO (prior } \\
\text { sterilization) }\end{array}$ & $\begin{array}{l}\mathrm{pg} / \mathrm{mL} \\
\text { a) } 2.555 \pm 219 \\
\text { b) } 2.366 \pm 282 \\
\text { c) } 2.925 \pm 259 \\
\text { d) } 2.479 \pm 281\end{array}$ & ns & $\begin{array}{l}\text { a) } 9.5 \pm 0.2 \\
\text { b) } 10.1 \pm 0.4 \\
\text { c) } 9.8 \pm 0.2 \\
\text { d) } 9.3 \pm 0.3\end{array}$ & ns & $\begin{array}{l}\text { a) } 16.2 \pm 1.2 \\
\text { b) } 14.4 \pm 1.8 \\
\text { c) } 17.5 \pm 1.8 \\
\text { d) } 12.2 \pm 1.3\end{array}$ & ns & $n \cdot r$ & & $\begin{array}{l}\text { a) } 2.79 \pm 0.2 \\
\text { b) } 3.5 \pm 0.4 \\
\text { c) } 3.2 \pm 0.2 \\
\text { d) } 3.0 \pm 0.3\end{array}$ & ns & $n . r$ & - & $\begin{array}{l}\text { a) } 57.1(16 / \\
\text { 28) } \\
\text { b) } 46.7(7 / \\
\text { 15) } \\
\text { c) } 52.9(18 / \\
34) \\
\text { d) } 58.3(7 / \\
\text { 12) }\end{array}$ & ns & $n \cdot r$ & - \\
\hline $\begin{array}{l}\text { Tal et al. } \\
{[12]}\end{array}$ & $\begin{array}{l}\text { a) } 26 \text { SLP (ECP) } \\
\text { Type: unilateral } \\
\text { b) } 52 \text { no surgery } \\
\text { (healthy subjects) }\end{array}$ & $\begin{array}{l}\text { pmol /L } \\
\text { a) } 5189 \pm 3310 \\
\text { b) } 5631 \pm 3512\end{array}$ & ns & $\begin{array}{l}\text { a) } 11.6 \pm 3.1 \\
\text { b) } 10.8 \pm 2.5\end{array}$ & ns & $\begin{array}{l}\text { a) } 8.6 \pm 5.3 \\
\text { b) } 8.4 \pm 4.9\end{array}$ & ns & $\begin{array}{l}\text { a) } 5.5 \pm 3.4 \\
\text { b) } 4.0 \pm 2.3\end{array}$ & ns & $n . r$ & - & $n \cdot r$ & - & $n \cdot r$ & - & $n \cdot r$ & - \\
\hline $\begin{array}{l}\text { Gelbaya et } \\
\text { al. [10] }\end{array}$ & $\begin{array}{l}\text { a) } 40 \text { SLP (HY) } \\
\text { Type: } 16 \text { unilateral, } 24 \\
\text { bilateral } \\
\text { b) } 25 \text { PTD } \\
\text { Type: } 9 \text { unilateral, } 16 \\
\text { bilateral } \\
\text { c) } 103 \text { no surgery } \\
\text { (TF, no HY) }\end{array}$ & $\begin{array}{l}\text { pmol/L } \\
\text { a) } 8558 \pm \\
4337.98 \\
\text { b) } 11192.7 \pm \\
4167.3 \\
\text { c) } 9512.78 \pm \\
4173.9\end{array}$ & .05 & $\begin{array}{l}\text { a) } 10.35 \pm 1.92 \\
\text { b) } 10.28 \pm 1.02 \\
\text { c) } 10.17 \pm 1.37\end{array}$ & ns & $\begin{array}{l}\text { a) } 10.23 \pm 6.08 \\
\text { b) } 13.68 \pm 5.17 \\
\text { c) } 12.92 \pm 8.75\end{array}$ & $\begin{array}{l}.05 \\
a \\
\text { vs } \\
b\end{array}$ & $\begin{array}{l}\text { a) } 6.78 \pm 4.58 \\
\text { b) } 8.52 \pm 4.75 \\
\text { c) } 7.80 \pm 5.48\end{array}$ & ns & n.r & & $\begin{array}{l}\text { a) } 18.2(10 / \\
55) \\
\text { b) } 12.8(5 / 39) \\
\text { c) } 11(18 / \\
\text { 163) }\end{array}$ & ns & $\begin{array}{l}\text { a) } 17.5(7 / \\
\text { 40) } \\
\text { b) } 20(5 / 25) \\
\text { c) } 16.5(17 / \\
103)\end{array}$ & ns & $\begin{array}{l}\text { a) } 17.5(7 / 40) \\
\text { b) } 16.0(4) \\
\text { c) } 13.6(14)\end{array}$ & ns \\
\hline $\begin{array}{l}\text { Moshin \& } \\
\text { Hotineanu } \\
\text { [11] }\end{array}$ & $\begin{array}{l}\text { a) } 60 \mathrm{SLP}(\mathrm{HY}) \\
\text { b) } 78 \mathrm{POT}(\mathrm{HY}) \\
\text { c) } 66 \text { no surgery }(\mathrm{HY}) \\
\text { type: not specified }\end{array}$ & $n \cdot r$ & - & $n \cdot r$ & - & $\begin{array}{l}\text { a) } 10.4 \pm 6.0 \\
\text { b) } 10.2 \pm 5.7 \\
\text { c) } 9.8 \pm 5.5\end{array}$ & ns & $\begin{array}{l}\text { a) } 7.0 \pm 4.7 \\
\text { b) } 6.9 \pm 4.6 \\
\text { c) } 6.8 \pm 4.6\end{array}$ & ns & $\begin{array}{l}\text { a) } 3.4 \pm 1.2 \\
\text { b) } 3.4 \pm 1.3 \\
\text { c) } 3.5 \pm 1.3\end{array}$ & ns & $n . r$ & - & $\begin{array}{l}\text { a) } 38(23 / 60) \\
\text { b) } 40(31 / 78) \\
\text { c) } 12(8 / 66)\end{array}$ & $\begin{array}{l}.05 \\
a, b \\
\text { vs C }\end{array}$ & $n \cdot r$ & - \\
\hline $\begin{array}{l}\text { Kontoravdis } \\
\text { et al. [9] }\end{array}$ & $\begin{array}{l}\text { a) } 50 \text { POT (HY) } \\
\text { b) } 50 \mathrm{SLP}(\mathrm{HY}) \\
\text { c) } 15 \text { no surgery (HY) } \\
\text { type: not specified }\end{array}$ & n.r. & & $\begin{array}{l}\text { a) } 12.3 \pm 2.4 \\
\text { b) } 11.9 \pm 2.5 \\
\text { c) } 13 \pm 1.9\end{array}$ & ns & $\begin{array}{l}\text { a) } 11.6 \pm 4.9 \\
\text { b) } 12.1 \pm 5.0 \\
\text { c) } 10.9 \pm 5.1\end{array}$ & ns & $\begin{array}{l}\text { a) } 8.7 \pm 3.9 \\
\text { b) } 8.53 \pm 4.0 \\
\text { c) } 7.9 \pm 5.1\end{array}$ & ns & $\begin{array}{l}\text { a) } 2.6 \pm 0.6 \\
\text { b) } 2.6 \pm 0.6 \\
\text { c) } 2.6 \pm 0.8\end{array}$ & ns & $\begin{array}{l}\text { a) } 19.5 \\
\text { b) } 24.8 \\
\text { c) } 5.6\end{array}$ & $\begin{array}{l}.03 \\
a \text { vs } \\
c \\
.007 \\
b \text { vs } \\
c\end{array}$ & $\begin{array}{l}\text { a) } 44.4 \\
\text { b) } 55.3 \\
\text { c) } 14.3\end{array}$ & $\begin{array}{l}.04 \\
a \text { vs } \\
c \\
.007 \\
b \text { vs } \\
c\end{array}$ & $\begin{array}{l}\text { a) } 37.8 \\
\text { b) } 48.9 \\
\text { c) } 7.1\end{array}$ & $\begin{array}{l}.03 \\
a \text { vs } \\
c \\
.004 \\
b \text { vs } \\
c\end{array}$ \\
\hline
\end{tabular}


Table 3 IVF outcome of patients included in the review (Continued)

\begin{tabular}{|c|c|c|c|c|c|c|c|c|c|c|c|c|c|c|c|c|c|}
\hline $\begin{array}{l}\text { Nakagawa } \\
\text { et al. [17] }\end{array}$ & $\begin{array}{l}\text { a) } 11 \text { PTD (HY) } \\
\text { b) } 6 \mathrm{SLP}(\mathrm{HY}) \\
\text { type: not specified }\end{array}$ & $\begin{array}{l}\mathrm{pg} / \mathrm{mL} \\
\text { a) } 1553 \pm 1468 \\
\text { b) } 1530 \pm 896\end{array}$ & ns & $n \cdot r$ & - & $\begin{array}{l}\text { a) } 5.3 \pm 4.7 \\
\text { b) } 7.5 \pm 5.5\end{array}$ & ns & $n . r$ & -1 & $n \cdot r$ & - & $n . r$ & - & $\begin{array}{l}\text { a) } 45.5 \\
\text { b) } 50.0\end{array}$ & ns & $n \cdot r$ & - \\
\hline $\begin{array}{l}\text { Almog et al. } \\
{[19]}\end{array}$ & $\begin{array}{l}\text { Before surgery } \\
\text { (HY, ECP) } \\
\text { a) } 36 \mathrm{SLP}(22 \\
\text { unilateral, } 14 \text { bilateral) } \\
\text { After Surgery } \\
\text { a) } 36 \mathrm{SLP}\end{array}$ & $\begin{array}{l}\mathrm{pg} / \mathrm{mL} \\
\text { Before surgery } \\
\text { a) } 1899.9 \pm 185 \\
\text { After Surgery } \\
\text { a) } 1997 \pm 231\end{array}$ & ns & $\begin{array}{l}\text { Before surgery } \\
\text { a) } 10.5 \pm 0.6 \\
\text { After Surgery } \\
\text { a) } 10.4 \pm 0.4\end{array}$ & ns & $\begin{array}{l}\text { Before surgery } \\
\text { a) } 10.2 \pm 6.6 \\
\text { After Surgery } \\
\text { a) } 10.3 \pm 7.4\end{array}$ & ns & $n . r$ & -1 & $n \cdot r$ & - & $n . r$ & - & $n \cdot r$ & - & $n \cdot r$ & - \\
\hline $\begin{array}{l}\text { Orvieto et } \\
\text { al. [18] }\end{array}$ & $\begin{array}{l}\text { Before surgery (HY) } \\
\text { a) } 15 \text { uni/bilateral } \\
\text { After surgery } \\
\text { b) } 15 \text { uni/bilateral }\end{array}$ & $\begin{array}{l}\mathrm{pg} / \mathrm{mL} \\
\text { a) } 1,996 \pm 885 \\
\text { b) 2,020 } \pm 981\end{array}$ & ns & $\begin{array}{l}\text { b) } 10.5 \pm 1.7 \\
\text { a) } 10.8 \pm 1.5\end{array}$ & ns & $\begin{array}{l}\text { a) } 11.6 \pm 5.9 \\
\text { b) } 10.2 \pm 6.1\end{array}$ & ns & $n . r$ & - & $\begin{array}{l}\text { a) } 2.7 \pm 1.1 \\
\text { b) } 2.3 \pm 0.7\end{array}$ & ns & & & $\begin{array}{l}\text { a) } 6.7(1 / 15) \\
\text { b) } 40(6 / 15)\end{array}$ & .05 & $n . r$ & - \\
\hline Xi et al. [20] & $\begin{array}{l}\text { Before surgery (ECP) } \\
\text { a) } 76 \text { SLP } \\
-44 \text { unilateral } \\
\text { - } 32 \text { bilateral } \\
\text { After Surgery } \\
\text { a) } 76 \text { SLP } \\
\text { - } 44 \text { unilateral } \\
\text { - } 32 \text { bilateral } \\
\text { b) } 80 \text { no surgery } \\
\text { (healthy subjects) }\end{array}$ & $\begin{array}{l}\mathrm{pg} / \mathrm{mL} \\
\text { Before surgery } \\
\text { a) } 2663.5 \pm 1246 \\
2446.9 \pm 983.8^{\circ} \\
2512.5 \pm 1119.4 \\
\text { After surgery } \\
\text { a) } 2783 \pm 1281.3 \\
2860.8 \pm 1509.7 \\
2585 \pm 1216.2 \\
\text { b) } 2934.8 \pm \\
\text { 1234.9 }\end{array}$ & ns & $\begin{array}{l}\text { Before surgery } \\
\text { a) } 10.7 \pm 1.5 \\
11.1 \pm 1.5 \\
10.7 \pm 1.6 \\
\text { After surgery: } \\
\text { a) } 11.1 \pm 1.8 \\
11 \pm 1.3 \\
10.8 \pm 1.9 \\
\text { b) } 11.1 \pm 1.8\end{array}$ & ns & $\begin{array}{l}\text { Before surgery } \\
\text { a) } 11.1 \pm 5.4 \\
11.3 \pm 5.1 \\
11.9 \pm 6.0 \\
\text { After surgery } \\
\text { a) } 11.6 \pm 4.1 \\
11.1 \pm 4.3 \\
11.9 \pm 5.5 \\
\text { b) } 11.5 \pm 4.4\end{array}$ & ns & $\begin{array}{l}\text { Before } \\
\text { surgery } \\
\text { a) } 8.3 \pm 4.4 \\
7.5 \pm 2.9 \\
8.2 \pm 4.3 \\
\text { After surgery } \\
\text { a) } 8.4 \pm 3.9 \\
8.4 \pm 4.0 \\
8.4 \pm 3.4 \\
\text { b) } 8.0 \pm 3.1\end{array}$ & ns & $n \cdot r$ & - & $n . r$ & - & $n . r$ & - & $n . r$ & - \\
\hline $\begin{array}{l}\mathrm{Na} \text { et al. } \\
\text { [21] }\end{array}$ & $\begin{array}{l}\text { a) } 41 \mathrm{SLP} \\
\text { b) } 56 \text { sclerotherapy } \\
\text { type: not specified }\end{array}$ & $n . r$ & - & $n . r$ & - & $\begin{array}{l}\text { a) } 6.2 \pm 1.0 \\
\text { b) } 12.1 \pm 11\end{array}$ & ns & $n . r$ & -1 & $n \cdot r$ & - & $n . r$ & - & $\begin{array}{l}\text { a) } 40(17 / 43) \\
\text { b) } 38(23 / 60)\end{array}$ & ns & $n \cdot r$ & - \\
\hline $\begin{array}{l}\text { Lin et al. } \\
{[24]}\end{array}$ & $\begin{array}{l}\text { a) } 103 \text { SLP (HY, ECP) } \\
\text { b) } 185 \text { (prior } \\
\text { sterilization, } \\
\text { tuboplasty, PTO) } \\
\text { type: not specified }\end{array}$ & $\begin{array}{l}\mathrm{pg} / \mathrm{mL} \\
\text { a) } 2153 \pm 1239 \\
\text { b) } 2340 \pm 1529\end{array}$ & ns & $\begin{array}{l}\text { a) } 8.8 \pm 1.4 \\
\text { b) } 8.8 \pm 1.5\end{array}$ & ns & $\begin{array}{l}\text { a) } 7.4 \pm 3.9 \\
\text { b) } 7.6 \pm 4.1\end{array}$ & $n s$ & $n . r$ & - & $\begin{array}{l}\text { a) } 2.5 \pm 0.8 \\
\text { b) } 2.5 \pm 0.8\end{array}$ & ns & $\begin{array}{l}\text { a) } 21.4(56 / \\
261) \\
\text { b) } 21.0(98 / \\
465)\end{array}$ & ns & $\begin{array}{l}\text { a) } 53.5(55 / \\
\text { 99) } \\
\text { b) } 43.5(77 / \\
\text { 177) }\end{array}$ & ns & $\begin{array}{l}\text { a) } 30.3(30 / 99) \\
\text { b) } 25.4(45 / 77)\end{array}$ & ns \\
\hline Ni et al. [22] & $\begin{array}{l}\text { a) } 26 \text { bilateral SLP } \\
\text { b) } 34 \text { unilateral SLP } \\
\text { c) } 23 \text { PTO } \\
\text { d) no surgery (TF, no } \\
\text { HY) }\end{array}$ & $n . r$ & - & $\begin{array}{l}\text { a) } 8.15 \pm 1.29 \\
\text { b) } 8.21 \pm 1.27 \\
\text { c) } 8.83 \pm 1.37 \\
\text { d) } 8.18 \pm 1.35\end{array}$ & ns & $\begin{array}{l}\text { a) } 9.15 \pm 3.73 \\
\text { b) } 11.59 \pm 6.14 \\
\text { c) } 10.70 \pm 4.92 \\
\text { d) } 10.82 \pm 4.82\end{array}$ & $n s$ & $\begin{array}{l}\text { a) } 6.04 \pm 2.85 \\
\text { b) } 7.74 \pm 4.23 \\
\text { c) } 7.22 \pm 3.25 \\
\text { d) } 7.57 \pm 3.74\end{array}$ & ns & $\begin{array}{l}\text { a) } 1.96 \pm 0.45 \\
\text { b) } 2.03 \pm 0.39 \\
\text { c) } 2.0 \pm 0.43 \\
\text { d) } 1.96 \pm 0.20\end{array}$ & ns & $\begin{array}{l}\text { a) } 51(26 / 51) \\
\text { b) } 30.4(21 / \\
69) \\
\text { c) } 39.1(18 / \\
\text { 46) } \\
\text { d) } 28(28 / \\
\text { 100) }\end{array}$ & & $\begin{array}{l}\text { a) } 65.4(17 / \\
\text { 26) } \\
\text { b) } 47.1(16 / \\
34) \\
\text { c) } 52.2(12 / \\
\text { 23) } \\
\text { d) } 49(25 / 51)\end{array}$ & ns & $\begin{array}{l}\text { LBR } \\
\text { a) } 61.5(16 / 26 \\
\text { b) } 41.2(14 / 34) \\
\text { c) } 52.2(12 / 23) \\
\text { d) } 45.1(23 / 51)\end{array}$ & ns \\
\hline $\begin{array}{l}\text { Grynnerup } \\
\text { et al. [25] }\end{array}$ & $\begin{array}{l}\text { a) } 16 \text { SLP (HY) } \\
\text { Type: (uni/bilateral) } \\
\text { not specified } \\
\text { b) } 42 \text { no surgery (TF, } \\
\text { with or without HY) } \\
\text { c) } 13 \text { no surgery } \\
\text { (unexplained infertility, } \\
\text { no HY) }\end{array}$ & $n . r$ & - & n.r & - & $\begin{array}{l}\text { a) } 7(3-31 \\
\text { range) } \\
\text { b) } 12(3-30) \\
\text { c) } 9(9-38)\end{array}$ & $\begin{array}{l}.05 \\
a \\
\text { vs } \\
b\end{array}$ & $n . r$ & - & $\begin{array}{l}\text { a) } 2(1-3) \\
\text { b) } 2(1-2) \\
\text { c) } 2(1-2)\end{array}$ & ns & $n . r$ & & $n . r$ & & $n . r$ & - \\
\hline
\end{tabular}


Table 3 IVF outcome of patients included in the review (Continued)

\begin{tabular}{|c|c|c|c|c|c|c|c|c|c|c|c|c|c|c|c|c|c|}
\hline $\begin{array}{l}\text { Hill et al. } \\
\text { [27] }\end{array}$ & $\begin{array}{l}\text { Surgery Group (ECP) } \\
\text { a) } 36 \text { unilateral SLP } \\
\text { - pre-surgery } \\
\text { - post-surgery } \\
\text { Medical Group (ECP) } \\
\text { b) } 153 \text { methotrexate } \\
\text { - pre-treatment } \\
\text { - post-treatment }\end{array}$ & $n \cdot r$ & - & $n \cdot r$ & - & $\begin{array}{l}\text { a) }-13(3-31) \\
-12(3-31) \\
\text { b) }-14(2-36) \\
-14(0-35)\end{array}$ & ns & $n \cdot r$ & - & n.r & - & $n \cdot r$ & - & $\begin{array}{l}\text { a) } 14 \text { (39) } \\
\text { b) } 68(44)\end{array}$ & ns & $\begin{array}{l}\text { a) } 13(33) \\
\text { b) } 53 \text { (35) }\end{array}$ & ns \\
\hline $\begin{array}{l}\text { Pereira et al. } \\
{[29]}\end{array}$ & $\begin{array}{l}\text { a) } 107 \mathrm{MTX}(\mathrm{ECP}) \rightarrow 88 \\
\text { IVF } \\
\text { b) } 37 \text { unilateral SLP } \\
(\mathrm{ECP}) \rightarrow 22 \text { IVF }\end{array}$ & $n \cdot r$ & - & $\begin{array}{l}\text { a) MTX } \\
\text { pre } 9.55 \pm 1.99 \\
\text { post } 9.76 \pm 2.33 \\
\text { b) SLP } \\
\text { pre } 9.63 \pm 2.21 \\
\text { post } 9.86 \pm 1.93\end{array}$ & $\begin{array}{l}\text { a) } \\
\text { ns } \\
\text { b) } \\
\text { ns }\end{array}$ & $\begin{array}{l}\text { a) MTX } \\
\text { pre } 12.4 \pm 5.77 \\
\text { post } 10.6 \pm \\
5.51 \\
\text { b) SLP } \\
\text { pre } 12.2 \pm 6.43 \\
\text { post } 10.2 \pm \\
4.23\end{array}$ & $\begin{array}{l}\text { a) } \\
.03 \\
\text { b) } \\
\text { ns }\end{array}$ & $n \cdot r$ & - & $\begin{array}{l}\text { a) MTX } \\
\text { pre } 2.92 \pm 1.24 \\
\text { post } 2.95 \pm 1.38 \\
\text { b) SLP } \\
\text { pre } 2.86 \pm 1.19 \\
\text { post } 2.81 \pm 1.01\end{array}$ & $\begin{array}{l}\text { a) } \\
\text { ns } \\
\text { b) } \\
\text { ns }\end{array}$ & $n \cdot r$ & - & $n \cdot r$ & - & $\begin{array}{l}\text { LBR } \\
\text { a) MTX } \\
\text { post } 32.95 \text { (29) } \\
\text { b) SLP } \\
\text { post } 36.3 \text { (8) }\end{array}$ & \\
\hline Ye et al. [28] & $\begin{array}{l}\text { a) } 83 \text { unilateral SLP } \\
\text { b) } 41 \text { bilateral SLP } \\
\text { c) } 74 \text { no surgery }\end{array}$ & $\begin{array}{l}\mathrm{pg} / \mathrm{mL} \\
\text { a) } 38.3 \pm 14.91 \\
\text { b) } 41.41 \pm 16.59 \\
\text { c) } 36.49 \pm 16.77\end{array}$ & ns & $\begin{array}{l}\text { a) } 9.6 \pm 1.76 \\
\text { b) } 9.39 \pm 2.12 \\
\text { c) } 9.78 \pm 1.62\end{array}$ & ns & $\begin{array}{l}\text { a) } 7.83 \pm 4.16 \\
\text { b) } 6.98 \pm 4.15 \\
\text { c) } 8.42 \pm 4.04\end{array}$ & ns & $\begin{array}{l}\text { a) } 3.39 \pm 3.03 \\
\text { b) } 3.15 \pm 2.51 \\
\text { c) } 3.5 \pm 2.6\end{array}$ & ns & $n . r$ & - & $n . r$ & - & n.r & - & $n . r$ & - \\
\hline $\begin{array}{l}\text { Odesjo et } \\
\text { al. [30] }\end{array}$ & $\begin{array}{l}\text { a) } 118 \text { unilateral SLP } \\
\text { b) } 35 \text { unilateral } \\
\text { salpingotomy }\end{array}$ & $n \cdot r$ & - & $n \cdot r$ & - & $\begin{array}{l}\text { a) } 11.69 \pm 5.59 \\
\text { b) } 11.8 \pm 6.1\end{array}$ & ns & $n \cdot r$ & - & $\begin{array}{l}\text { a) } 104(88 \%) \\
\text { b) } 30(85.7 \%)\end{array}$ & ns & $n \cdot r$ & - & $\begin{array}{l}\text { a) } 32 \\
\text { (27.1\%) } \\
\text { b) } 8(22.9 \%)\end{array}$ & ns & $\begin{array}{l}\text { LBR } \\
\text { a) } 21(17.8) \\
\text { b) } 7(20)\end{array}$ & ns \\
\hline
\end{tabular}

SLP, salpingectomy; n.r, not reported; n.s, not significant; ECP, ectopic pregnancy; HY, Hydrosalpinx; ECP, ectopic pregnancy; TOA, tubo-ovarian abscess; TF, tubal factor; LPS, laparoscopy; LPT, laparotomy; PTD, proximal tubal division; PTO, proximal tubal occlusion; MTX, methotrexate; LBR, live birth rate; IVF, in-vitro fertilization 
laparotomy salpingectomy with the non-treated site in the same cohort assigned as controls.

Concerning AMH serum values after uni/bilateral salpingectomy a single manuscript reported evidence evaluating $\mathrm{AMH}$ before and after the surgical procedure in the same cohort of patients. Venturella et al. [31], in a sample of 91 and 95 patients treated by unilateral salpingectomy using standard and wide excision approach respectively, did not observe statistically significant differences in terms of decrease in $\mathrm{AMH}$ prior to and 3 months after surgical intervention. Three papers [22, $25,28]$ reported evidence from observational studies comparing surgically treated patients versus controls. Two of the above mentioned papers compared a total of 76 cases of uni/bilateral salpingectomy versus 23 tubal ligations versus 106 non surgically treated patients and found no significant difference in terms of $\mathrm{AMH}$ $[22,25]$. Only one paper comparing a total of 124 cases of uni/bilateral salpingectomy and 74 untreated controls found a significant decrease in $\mathrm{AMH}$ value in the treated group [28]. Finally, Findley et al. [26] in a cohort of 30 patients undergoing total hysterectomy (15 bilateral salpingectomies vs. 15 untreated patients) did not find significant differences in terms of AMH decrease neither within the same group, nor between the two study groups

\section{Ovarian reserve markers after unilateral vs. bilateral salpingectomy}

Only three papers $[20,22,28]$ evaluated the impact of the type of surgical procedure by comparing unilateral vs. bilateral salpingectomy. $\mathrm{Ni}$ et al. [22] comparing 26 bilateral salpingectomies, 34 unilateral salpingectomies and 51 untreated controls found no significant differences, although in the bilateral salpingectomy group, a slightly inferior $\mathrm{AMH}$ in terms of absolute value was observed. Also Xi et al. [20] in an observational study which considered exclusively FSH levels (44 unilateral vs. 32 bilateral salpingectomy before and after surgery, and 80 untreated controls) observed no significant differences between groups. On the contrary, Ye et al. [28], analyzed 83 unilateral vs. 41 bilateral salpingectomy and 74 untreated patients, and found a significant decrease in both AMH and AFC and a significant increase in FSH in treated patients. This trend was markedly pronounced in patients treated by bilateral salpingectomy.

\section{Effects on ART outcomes}

A total of 24 studies for a total of 3001 patients have reported data on the effects of salpingectomy on ART outcomes. Of these 1,334 were treated by uni/bilateral salpingectomy, 519 (38.9\%) patients underwent unilateral salpingectomy, 346 (25.94\%) bilateral salpingectomy and 469 (35.16 \%) unspecified. The principal indication for the surgery was a previous ectopic pregnancy/ies and hydrosalpinx in $7[1,5,12,20,27,29,30]$ and 13 studies [2-4, $6-11,17,18,21,25]$, respectively. In 4 studies, both patients treated for ectopic pregnancy and hydrosalpinx were included in the analysis [19, 22, 24, 28].

\section{Length of stimulation}

All 16 papers which analyzed the length of stimulation, defined as number of days of gonadotropin administration, found a non-statistically significant different between salpingectomy vs. non-salpingectomy patients $[1-10,12$, 18-20, 28, 29].

\section{$E_{2}$ levels}

Plasmatic $\mathrm{E}_{2}$ levels on the day of human chorionic gonadotropin (hCG) administration (for ovulation triggering) resulted nonsignificant different between salpingectomy and non-salpingectomy arms in 10 studies $[1,2,8,12,17-20$, $24,28]$. In only one retrospective analysis, including 40 patients treated by salpingectomy due to hydrosalpinx compared to 103 patients with tubal factor infertility (no hydrosalpinx), reported a significant reduction in $\mathrm{E}_{2}$ levels in the salpingectomy group [10].

\section{Oocytes retrieved}

A total of 21 studies described the absence of any significant difference in terms of number of oocytes retrieved between salpingectomy and non-salpingectomy patients or in pre and post-surgical procedure in the same cohort of women [2-4, 6-12, 17-22, 24, 27, 28, 30]. Lass et al. [1] compared 29 patients treated by salpingectomy for ectopic pregnancy to 73 healthy women reporting a significant reduction in oocyte yield in the ipsilateral compared to the contralateral ovary. Grynnerup et al. [25] compared a total of 16 cases of hydrosalpinx treated by salpingectomy compared to 42 patients affected by tubal factor infertility (with or without hydrosalpinx) managed conservatively. A significant reduction of oocyte yield in the treated group was observed [25].

\section{Embryos obtained and/or transferred}

All $11[2,4,6,7,9-12,20,22,28]$ and 14 [1, 3-5, 7-9, 11, $18,22,24,25,29,30]$ studies which analyzed, respectively, the number of embryos obtained and of embryos transferred found no statistically significant difference between salpingectomy and non-salpingectomy patients.

\section{Implantation rate}

Only 6 studies assessed the implantation rate after IVF and embryo transfer. Two randomized controlled trials (RCTs) which compared patients with HY treated surgically or conservatively before IVF clearly demonstrated a significant improvement in implantation rates [4, 9]. This improvement is even more prominent in ultrasound 
visible hydrosalpinx [4]. One RCT and one retrospective study found no significant differences between treated and untreated women, even if surgically treated patients for hydrosalpinx showed a better implantation rate in terms of absolute value compared to untreated subjects $[2,10]$. Two further retrospective studies found no significant improvement of implantation rate in patients after salpingectomy; however, in these cases, the control subjects were represented by patients with tubal factor infertility without hydrosalpinx $[3,24]$.

\section{Clinical pregnancy rate}

Clinical pregnancy rate was reported as outcome measure in 16 studies. In particular, five RCTs compared a total of 359 patients affected by hydrosalpinx treated by salpingectomy vs. 281 untreated control subjects [2, 4, $7,9,11]$. Four of these studies reported a clear significant improvement in the pregnancy rate of treated vs. untreated subjects $[4,7,9,11]$. The remaining RCT did not show a significant improvement in pregnancy rate, even if a better result in terms of absolute value for transfer cycle in the salpingectomy group was reported [2]. All other observational and retrospective studies, with the exception of that reported by Orvieto et al. [18], did not report differences in terms of pregnancy rate in the salpingectomy compared to the control group; however, patients in the control group were generally affected by infertility for tubal factor without hydrosalpinx or were patients who underwent a surgical or medical procedure other than salpingectomy $[1,3,8,10,17,18,21$, $22,24,27,30]$.

\section{Ongoing pregnancy/live-birth rate}

Data on ongoing pregnancy and the live-birth rates were reported in 11 studies. Four RCTs compared a total 299 patients affected by hydrosalpinx treated by salpingectomy vs. 215 untreated controls [2, 4, 7, 9]. In 3 of these studies [4, 7, 9], a significant improvement in ongoing pregnancy rate/live birth rate in treated vs. untreated subjects was observed. A further RCT did not show significant improvement of pregnancy rate, even if a better result in terms of absolute value for transfer cycle after salpingectomy was reported [2]. The other available studies reported an improvement after salpingectomy group; however, they were non-randomized and poorly controlled. In fact, the controls were generally affected by infertility for tubal factor without hydrosalpinx or were patients who underwent surgical or medical procedures other than salpingectomy $[3,10$, $22,24,27,29,30]$.

\section{Discussion}

During the past decades many debates have been raised concerning the potential detrimental effects of hydrosalpinx (both unilateral and bilateral) on implantation and pregnancy rate after ART [32]. Two meta-analyses, published at the end of the nineties, were developed with the aim to definitively clarify this topic. The first one, analyzing more than 6,700 IVF cycles from 11 studies demonstrated a pregnancy rate of less than $49 \%$ and a miscarriage rate $2-3$ fold greater in patients with hydrosalpinx compared to controls subjects with tubal factor infertility without hydrosalpinx [odds ratio (OR 50.7), $95 \%$ confidence interval (CI) 41.4 to 62.2) [33]. The second one, involving a total of 5,592 women (including many of the same studies used in the first metaanalysis), showed a delivery rate per cycle of $13.4 \%$ vs. $23.4 \%$ (OR 0.58 , 95\%CI 0.49 to 0.69 ) for hydrosalpinx group and non- hydrosalpinx group, respectively [34].

In view of the above evidence it seemed logical to propose surgery prior to ART in order to avert possible deleterious effects derived from tubal fluids and consequently increase their success rate [4]. Laparoscopic unilateral or bilateral salpingectomy has been the first and most studied surgical technique proposed. However, although several observational and retrospective studies have been published, only five RCTs (of which one published only in abstract form) concerning the potential benefit of prophylactic laparoscopic salpingectomy before ART in case of hydrosalpinx were conducted. Despite potential bias due to small sample size and lack of blind randomization, (see Table 1 for more details) data analysis in all trials showed a clear advantage in terms of implantation rate, pregnancy rate and ongoing pregnancy rate in treated patients compared to untreated controls $[2,4,6,7,9,11]$. The derived data encouraged the scientific community to recommend tubal removal or tubal occlusion for hydrosalpinx prior to ART [14, 15, 32, 35]. Though these recommendations may be of use in the management of evident hydrosalpinx, controversy persists regarding the ideal management of smaller unilateral or bilateral hydrosalpinx due to the potential detrimental effects of surgery on the ovarian reserve. Analogous to the current trend in surgical management of patients affected by ovarian endometriomas $[36,37]$ tubal surgery before ART treatments is an ongoing debate.

With the intent of proposing a solution to the current dilemma, we analyzed several studies aimed at quantifying possible damage on ovarian reserve caused by salpingectomy. Considering data from operated vs. non-operated site, almost all studies, with the exception of Dar' data [5], reported a significant reduction of AFC and number of oocytes retrieved after ovarian stimulation limited to the ipsilateral ovary $[1,13,18,25]$.

The pathophysiological explanation of this finding may be related to two important phenomena: surgical dissection of vascular supply (mesosalpinx vessels) and energy 
spread by monopolar or bipolar devices [13, 18, 31, 38]. It is important to remember that AFC is only a surrogate of ovarian reserve that should be interpreted ideally in association with basal AMH, FSH and chronological age [39-41]. Trials investigating variations in ovarian reserve determined by the combination of both AFC and biochemical assay of FSH and AMH levels seems to suggest a non-significant reduction in the cohort of treated patients compared to controls $[8,16,17,20-23,25-27,31]$.

This finding is likely affected by the bias of considering unilateral salpingectomy (in which the damage may happen) while the estimated ovarian reserve considers both ovaries. It is fundamental to understand the implications of bilateral vs. unilateral salpingectomy on ovarian reserve and potential response to controlled stimulation. Data regarding this last point are scarce and only three studies analyzed this topic $[20,22,28]$. In only one study [28] a significant decrease in $\mathrm{AMH}$ and an increase in $\mathrm{FSH}$ serum values in the bilateral salpingectomy group was detected, even if after ovarian stimulation there was no difference in the number of oocytes collected. Interestingly, although all other Authors did not observe significant differences, worse outcomes in terms of absolute value were reported in bilateral salpingectomy group [22, 28].

Available data is scarce and does not clarify the issue of the effect of potential additional damage caused by bilateral as opposed to unilateral salpingectomy. The lack of clear evidence does not permit speculation regarding the potential benefit of prophylactic salpingectomy in patients undergoing ART and affected by unilateral hydrosalpinx and contralateral or bilateral tubal occlusion without hydrosalpinx. Certainly, if data reported in the last trial by Venturella et al. [31] suggesting no detrimental effect on ovarian reserve in bilateral salpingectomy will be confirmed by further trials, counselling for surgery in patients affected by tubal occlusion (also in cases without hydrosalpinx) may have a rationale and may result in further improvements of ART success rates (avoiding implantation failure or even more important tubal pregnancy). Indeed, when considering as outcome measure the implantation rate, pregnancy rate and ongoing/live-birth rate after ART in patients with hydrosalpinx, data from four RCTs suggest distinct improvements in patients treated by salpingectomy $[4,6,7,9,11]$.

Infertile women affected by hydrosalpinx are at increased risk for poor outcomes after IVF for a variety of reasons linked to chronic subclinical infections, inflammation and endometritis [42-44]. The pathophysiological explanation may be related to the possible accumulation of hydrosalpinx fluid within the endometrial cavity, which may have detrimental effects on endometrial receptivity and embryo development by a direct embryotoxic effect, mechanical washing of the blastocyst and by the dilatational effect of hydrosalpinx fluids on essential nutrients and fluids [45-52]. Moreover, although the data from these RCTs would apparently put an end to the controversy, the fact that the size and the localization (unilateral versus bilateral) of the hydrosalpinx recommend caution in the interpretation of data. The final aim of our review is non only to offer an overview of available data on this topic but to invite both scientists and clinicians in generating new data in order to finally clarify the role of salpingectomy on ovarian reserve. Indeed, just as for example, from our manuscript submission to final acceptance by the journal, 2 meta-analysis have been published in a single journal (August and October 2016) reporting opposite results and potentially generating more confusion $[53,54]$.

\section{Conclusions}

From the analysis of all available literature it is possible to conclude that in the presence of tubal disease a surgical approach based on unilateral salpingectomy may be considered safe and without negative effects on ovarian reserve markers and on ovarian response to ovarian stimulation protocols. Further trials aimed at confirming both the positive effects of tubal surgery before ART and the safety of bilateral salpingectomy are necessary to definitively state when and why unilateral rather than bilateral salpingectomy are recommended in cases of bilateral tubal blockage with or without hydrosalpinx.

\section{Acknowledgements}

No personal acknowledgement is declared.

Funding

No specific funding was sought for the study.

Availability of data and materials

Please contact author for data requests.

Authors' contributions

All authors interpreted data, revised the manuscript for intellectual content, drafted the manuscript and approved its final version.

Competing interests

No financial and non-financial competing interest involves the authors.

Consent for publication

Not applicable.

Ethics approval and consent to participate

Not applicable.

\section{Author details}

'Department of Woman and Child Health, University of Padua, Padua, Italy. ${ }^{2}$ Department of Obstetrics and Gynecology, NHS Trust - Northampton General Hospital, Northampton, United Kingdom. ${ }^{3}$ Unit of Reproductive Surgery and Medicine, ASMN-IRCCS of Reggio Emilia, Reggio Emilia, Italy.

Received: 19 September 2016 Accepted: 19 October 2016

Published online: 03 November 2016

References

1. Lass A, Ellenbogen A, Croucher C, Trew G, Margara R, Becattini C, Winston RM. Effect of salpingectomy on ovarian response to superovulation in an in vitro fertilization-embryo transfer program. Fertil Steril. 1998;70:1035-8. 
2. Déchaud H, Daurès JP, Arnal F, Humeau C, Hédon B. Does previous salpingectomy improve implantation and pregnancy rates in patients with severe tubal factor infertility who are undergoing in vitro fertilization? A pilot prospective randomized study. Fertil Steril. 1998;69:1020-5.

3. Bredkjaer HE, Ziebe S, Hamid B, Zhou Y, Loft A, Lindhard A, Andersen AN. Delivery rates after in-vitro fertilization following bilateral salpingectomy due to hydrosalpinges: a case control study. Hum Reprod. 1999;14:101-5.

4. Strandell A, Lindhard A, Waldenström U, Thorburn J, Janson PO, Hamberger L. Hydrosalpinx and IVF outcome: a prospective, randomized multicentre trial in Scandinavia on salpingectomy prior to IVF. Hum Reprod. 1999;14:2762-9.

5. Dar P, Sachs GS, Strassburger D, Bukovsky I, Arieli S. Ovarian function before and after salpingectomy in artificial reproductive technology patients. Hum Reprod. 2000;15:142-4.

6. Strandell A, Lindhard A, Waldenström U, Thorburn J. Prophylactic salpingectomy does not impair the ovarian response in IVF treatment. Hum Reprod. 2001;16:1135-9.

7. Strandell A, Lindhard A, Waldenström U, Thorburn J. Hydrosalpinx and IVF outcome: cumulative results after salpingectomy in a randomized controlled trial. Hum Reprod. 2001;16:2403-10

8. Surrey ES, Schoolcraft WB. Laparoscopic management of hydrosalpinges before in vitro fertilization-embryo transfer: salpingectomy versus proximal tubal occlusion. Fertil Steril. 2001;75:612-7.

9. Kontoravdis A, Makrakis E, Pantos K, Botsis D, Deligeoroglou E, Creatsas G. Proximal tubal occlusion and salpingectomy result in similar improvement in in vitro fertilization outcome in patients with hydrosalpinx. Fertil Steril. 2006;86:1642-9.

10. Gelbaya TA, Nardo LG, Fitzgerald CT, Horne G, Brison DR, Lieberman BA. Ovarian response to gonadotropins after laparoscopic salpingectomy or the division of fallopian tubes for hydrosalpinges. Fertil Steril. 2006:85:1464-8.

11. Moshin $V$, Hotineanu A. Reproductive outcome of the proximal tubal occlusion prior to IVF in patients with hydrosalpinx. Abstracts of the 22nd Annual Meeting of the ESHRE, Praque, Czech Republic, 18-21 June 2006.

12. Tal J, Paltieli Y, Korobotchka R, Ziskind G, Eibschitz I, Ohel G. Ovarian response to gonadotropin stimulation in repeated IVF cycles after unilateral salpingectomy. J Assist Reprod Genet. 2002;19:451-5.

13. Chan CC, Ng EH, Li CF, Ho PC. Impaired ovarian blood flow and reduced antral follicle count following laparoscopic salpingectomy for ectopic pregnancy. Hum Reprod. 2003;18:2175-80,

14. National Collaborating Centre for Women's and Children's Health (UK) Fertility: Assessment and Treatment for People with Fertility Problems. London: Royal College of Obstetricians \& Gynaecologists (UK); 2013.

15. Johnson N, van Voorst S, Sowter MC, Strandell A, Mol BW. Surgical treatment for tubal disease in women due to undergo in vitro fertilisation. Cochrane Database Syst Rev. 2010;1:CD002125.

16. Sezik M, Ozkaya O, Demir F, Sezik HT, Kaya H. Total salpingectomy during abdominal hysterectomy: effects on ovarian reserve and ovarian stromal blood flow. J Obstet Gynaecol Res. 2007;33:863-9.

17. Nakagawa K, Ohgi S, Nakashima A, Horikawa T, Irahara M, Saito H. Laparoscopic proximal tubal division can preserve ovarian reserve for infertility patients with hydrosalpinges. J Obstet Gynaecol Res. 2008;34:1037-42.

18. Orvieto R, Saar-Ryss B, Morgante G, Gemer O, Anteby EY, Meltcer S. Does salpingectomy affect the ipsilateral ovarian response to gonadotropin during in vitro fertilization-embryo transfer cycles? Fertil Steril. 2011:95:1842-4.

19. Almog B, Wagman I, Bibi G, Raz Y, Azem F, Groutz A, Barkan G, Holzer H, Amit A, Tulandi T, Levin I. Effects of salpingectomy on ovarian response in controlled ovarian hyperstimulation for in vitro fertilization: a reappraisal. Fertil Steril. 2011:95:2474-6.

20. Xi W, Gong F, Tang Y, Zhang H, Lu G. Ovarian response to gonadotropins after laparoscopic salpingectomy for ectopic pregnancy. Int J Gynaecol Obstet. 2012;116:93-6.

21. Na ED, Cha DH, Cho JH, Kim MK. Comparison of IVF-ET outcomes in patients with hydrosalpinx pretreated with either sclerotherapy or laparoscopic salpingectomy. Clin Exp Reprod Med. 2012;39:182-6.

22. Ni L, Sadiq S, Mao Y, Cui Y, Wang W, Liu J. Influence of various tubal surgeries to serum antimullerian hormone level and outcome of the subsequent IVF-ET treatment. Gynecol Endocrinol. 2013;29:345-9.

23. Uyar I, Yucel OU, Gezer C, Gulhan I, Karis B, Hanhan HM, Ozeren M. Effect of single-dose methotrexate on ovarian reserve in women with ectopic pregnancy. Fertil Steril. 2013;100:1310-3.
24. Lin YJ, Ou YC, Huang FJ, Lin PY, Kung FT, Lan KC. Ovarian response to gonadotropins in patients with tubal factor infertility: salpingectomy versus nonsalpingectomy. J Minim Invasive Gynecol. 2013;20:637-41.

25. Grynnerup AG, Lindhard A, Sørensen S. Anti-Müllerian hormone levels in salpingectomized compared with nonsalpingectomized women with tubal factor infertility and women with unexplained infertility. Acta Obstet Gynecol Scand. 2013;92:1297-303.

26. Findley AD, Siedhoff MT, Hobbs KA, Steege JF, Carey ET, MCCall CA, Steiner AZ. Short-term effects of salpingectomy during laparoscopic hysterectomy on ovarian reserve: a pilot randomized controlled trial. Fertil Steril. 2013;100:1704-8.

27. Hill MJ, Cooper JC, Levy G, Alford C, Richter KS, DeCherney AH, Katz CL, Levens ED, Wolff EF. Ovarian reserve and subsequent assisted reproduction outcomes after methotrexate therapy for ectopic pregnancy or pregnancy of unknown location. Fertil Steril. 2014;101:413-9.

28. Ye $X P$, Yang $Y Z$, Sun $X X$. A retrospective analysis of the effect of salpingectomy on serum anti-Müllerian hormone level and ovarian reserve. Am J Obstet Gynecol. 2015;212:53.e1-10.

29. Pereira N, Gerber D, Gerber RS, Lekovich JP, Elias RT, Spandorfer SD, Rosenwaks Z. Effect of methotrexate or salpingectomy for ectopic pregnancy on subsequent in vitro fertilization-embryo transfer outcomes. J Minim Invasive Gynecol. 2015;22:870-6.

30. Ödesjö E, Bergh C, Strandell A. Surgical methods for tubal pregnancy effects on ovarian response to controlled stimulation during IVF. Acta Obstet Gynecol Scand. 2015;94:1322-6.

31. Venturella R, Morelli M, Lico D, Di Cello A, Rocca M, Sacchinelli A, Mocciaro R, D'Alessandro P, Maiorana A, Gizzo S, Zullo F. Wide excision of soft tissues adjacent to the ovary and fallopian tube does not impair the ovarian reserve in women undergoing prophylactic bilateral salpingectomy: results from a randomized, controlled trial. Fertil Steril. 2015;104:1332-9.

32. Practice Committee of American Society for Reproductive Medicine in collaboration with Society of Reproductive Surgeons. Salpingectomy for hydrosalpinx prior to in vitro fertilization. Fertil Steril. 2008;90:66-8.

33. Zeyneloglu HB, Arici A, Olive DL. Adverse effects of hydrosalpinx on pregnancy rates after in vitro fertilization-embryo transfer. Fertil Steril. 1998;70:492-9.

34. Camus E, Poncelet C, Goffinet F, Wainer B, Merlet F, Nisand I, Philippe HJ. Pregnancy rates after in-vitro fertilization in cases of tubal infertility with and without hydrosalpinx: a meta-analysis of published comparative studies. Hum Reprod. 1999;14:1243-9.

35. Johnson N, van Voorst S, Sowter MC, Strandell A, Mol BW. Tubal surgery before IVF. Hum Reprod Update. 2011;17:3.

36. Gizzo S, Vitagliano A, Noventa M, Litta P, Saccardi C, Quaranta M. Surgery, endometriosis-related infertility and negative impact on ovarian reserve: "which came first, the hen or the egg?" An unresolved dilemma. Arch Gynecol Obstet. 2015;292:709-11.

37. Gizzo S, Conte L, Di Gangi S, Leggieri C, Quaranta M, Noventa M, Litta P, Saccardi C. Could surgeon's expertise resolve the debate about surgery effectiveness in treatment of endometriosis-related infertility? Arch Gynecol Obstet. 2015;292:217-23.

38. Litta P, Saccardi C, Gizzo S, Conte L, Ambrosi G, Sissi C, Palumbo M. Inflammatory cytokine expression following the use of bipolar electrocoagulation, ultracision harmonic scalpel and cold knife biopsy. Mol Med Rep. 2015;12:2985-90.

39. Gizzo S, Andrisani A, Esposito F, Oliva A, Zicchina C, Capuzzo D, Gangemi M, Nardelli GB. Ovarian reserve test: an impartial means to resolve the mismatch between chronological and biological age in the assessment of female reproductive chances. Reprod Sci. 2014;21:632-9.

40. Patrelli TS, Gizzo S, Sianesi N, Levati L, Pezzuto A, Ferrari B, Bacchi MA. Anti-Müllerian hormone serum values and ovarian reserve: can it predict a decrease in fertility after ovarian stimulation by ART cycles? PLOS One. 2012; $7:$ :44571.

41. Gizzo S, Andrisani A, Noventa M, Quaranta M, Esposito F, Armanini D, Gangemi M, Nardelli GB, Litta P, D'Antona D, Ambrosini G. Menstrual cycle length: a surrogate measure of reproductive health capable of improving the accuracy of biochemical/sonographical ovarian reserve test in estimating the reproductive chances of women referred to ART. Reprod Biol Endocrinol. 2015;13:28.

42. Copperman AB, Wells V, Luna M, Kalir T, Sandler B, Mukherjee T. Presence of hydrosalpinx correlated to endometrial inflammatory response in vivo. Fertil Steril. 2006;86:972-6. 
43. Patrelli TS, Franchi L, Gizzo S, Salvati MA, Berretta R, Piantelli G, Modena AB. Can the impact of pelvic inflammatory disease on fertility be prevented? Epidemiology, clinical features and surgical treatment: evolution over 8 years. J Reprod Med. 2013:58:425-33.

44. Franchi L, Patrelli TS, Berretta R, Rolla M, Gizzo S, Gramellini D, Bacchi Modena A, Nardelli GB. Role of D-dimer testing in severe pelvic inflammatory disease: a new usable marker to assess the need for fertility-impairing surgery? Fertil Steril. 2010;94:2372-5.

45. Lessey BA, Castelbaum AJ, Sawin SW, Sun J. Integrins as markers of uterine receptivity in women with primary unexplained infertility. Fertil Steril. 1995:63:535-42.

46. Meyer WR, Castelbaum AJ, Somkuti S, Sagoskin AW, Doyle M, Harris JE, Lessey BA. Hydrosalpinges adversely affect markers of endometrial receptivity. Hum Reprod. 1997;12:1393-8.

47. Cohen MA, Lindheim SR, Sauer MV. Hydrosalpinges adversely affect implantation in donor oocyte cycles. Hum Reprod. 1999;14:1087-9.

48. Mansour RT, Aboulghar MA, Serour Gl, Riad R. Fluid accumulation of the uterine cavity before embryo transfer: a possible hindrance for implantation. J In Vitro Fert Embryo Transf. 1991;8:157-9.

49. Sharara FI. The role of hydrosalpinx in IVF: simply mechanical? Hum Reprod. 1999;14:577-8.

50. Murray CA, Clarke HJ, Tulandi T, Tan SL. Inhibitory effect of human hydrosalpingeal fluid on mouse preimplantation embryonic development is significantly reduced by the addition of lactate. Hum Reprod. 1997;12:2504-7.

51. Murray DL, Sagoskin AW, Widra EA, Levy MJ. The adverse effect of hydrosalpinges on in vitro fertilization pregnancy rates and the benefit of surgical correction. Fertil Steril. 1998;69:41-5.

52. Koong MK, Jun JH, Song SJ, Lee HJ, Song IO, Kang IS. A second look at the embryotoxicity of hydrosalpingeal fluid: an in-vitro assessment in a murine model. Hum Reprod. 1998;13:2852-6.

53. Fan M, Ma L. Effect of salpingectomy on ovarian response to hyperstimulation during in vitro fertilization: a meta-analysis. Fertil Steril. 2016;106(2):322-329.e9.

54. Yoon SH, Lee JY, Kim SN, Chung HW, Park SY, Lee C. Does salpingectomy have a deleterious impact on ovarian response in in vitro fertilization cycles? Fertil Steril. 2016;106(5):1083-1092.e5.

\section{Submit your next manuscript to BioMed Central and we will help you at every step:}

- We accept pre-submission inquiries

- Our selector tool helps you to find the most relevant journal

- We provide round the clock customer support

- Convenient online submission

- Thorough peer review

- Inclusion in PubMed and all major indexing services

- Maximum visibility for your research

Submit your manuscript at www.biomedcentral.com/submit 